France-Lanord, C., Spiess, V., Klaus, A., Schwenk, T., and the Expedition 354 Scientists

Proceedings of the International Ocean Discovery Program Volume 354

publications.iodp.org

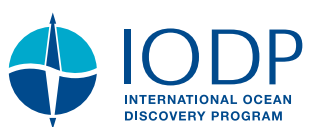

https://doi.org/10.14379/iodp.proc.354.202.2018

Check for updates

Contents

\title{
Data report: grain size analysis of Bengal Fan sediments at Sites U1450 and U1451, IODP Expedition 354 ${ }^{1}$
}

\author{
1 Abstract \\ 1 Introduction \\ 1 Methods \\ 2 Results \\ 11 Acknowledgments \\ 11 References
}

Swostik Kumar Adhikari, ${ }^{2,}{ }^{3}$ Tetsuya Sakai, ${ }^{3}$ and Kohki Yoshida ${ }^{4}$

Keywords: International Ocean Discovery Program, IODP, JOIDES Resolution, Expedition 354, Bengal Fan, Site U1450, Site U1451, grain size

\begin{abstract}
Grain size distributions of 311 sediment samples from Sites U1450 and U1451 of International Ocean Discovery Program (IODP) Expedition 354 were determined using laser diffraction. Most of the samples were from turbidites, but some hemipelagic beds were also examined. The mean grain size values show that siltsized particles are the dominant textural class, whereas the grain size values range from clay to coarse-grained sand. An overall upward change in mean grain size value reveals a slight coarseningupward trend. However, other parameters such as standard deviation, skewness, and kurtosis show no systematic relationship with depth in the holes. The analyzed samples cover the age range from recent to early Miocene. Shepard textural classification plots show the sediments are mostly sandy silts, silty sands, and clayey silts with a few silts and sands also present. Frequency curve plots of samples from individual turbidite beds show inversely graded beds are most common at Site U1450, whereas thicker massive beds are dominant at Site U1451.
\end{abstract}

\section{Introduction}

International Ocean Discovery Program (IODP) Expedition 354 to $8^{\circ} \mathrm{N}$ in the Bay of Bengal drilled at seven sites along a $320 \mathrm{~km}$ long transect across the Bengal Fan. The submarine fan in the study area consists of channel and levee complexes (see the Expedition 354 summary chapter; France-Lanord et al., 2016a). The evolution of the channel and levee complexes may be related to sea level changes, which are functions of external forces such as climatic changes and tectonics, in addition to autogenic processes such as shifting of submarine channels (Stow et al., 1985, 1996; Bouma et al., 1989; Bouma, 2000, 2004; Stelting et al., 2000). Grain size characteristics are one of the most fundamental features of clastic sediments (Folk, 1974; Shiki and Yamazaki, 1984; Pettijohn et al., 1987;
McManus, 1988, and others). Changes in grain size distribution are related to sedimentation processes, and such distributions can be expected to change even within a single channel-levee complex because of the growth of the levee through time (Gorsline, 1984; Manley et al., 1997; Bouma, 2000; Dennielou et al., 2006).

The sediments obtained during Expedition 354 consist mainly of turbidites. These turbidite sequences are generally separated by hemipelagic beds (mottled or bioturbated calcareous clays). The purpose of this study is to obtain grain size distributions of selected horizons of turbidites and their upward variation. Weight percentages of sand, silt, and clay yield statistical parameters of the resultant grain size distributions and hence provide comprehensive textural information for the sediments. This report presents the data set arising from grain size analysis of samples from two sites, U1450 and U1451. Most of the samples are turbidites, but data were also acquired for some hemipelagic beds. Two holes were drilled at each site, denoted Holes A and B. The deepest point of Hole U1450A was 687.4 meters below the seafloor (mbsf), and the depth of Hole U1450B ranged from 608 to 811.9 mbsf, which covers the age from recent to late Miocene. The lower limit of Hole U1451A was $582 \mathrm{mbsf}$, and the depth of Hole U1451B ranged from 542 to 1181 mbsf, which covers the age from recent to late Paleocene (see the Expedition 354 summary, Site U1450, and Site U1451 chapters, [France-Lanord et al., 2016a, 2016b, 2016c, respectively]).

\section{Methods}

\section{Sample preparation and analysis}

A total of 311 samples were collected from the two sites. Of these, 96 were taken from Hole U1450A, 24 from Hole U1450B, 100 from Hole U1451A, and 91 from Hole U1451B. Most of these sediment specimens were collected from the base (77 samples), middle (89 samples), and top (53 samples) horizons of the sandy and muddy (75 samples) parts of the well-preserved turbidites. Seventeen sedi-

\footnotetext{
${ }^{1}$ Adhikari, S.K., Sakai, T., Yoshida, K., 2018. Data report: grain size analysis of Bengal Fan sediments at Sites U1450 and U1451, IODP Expedition 354. In France-Lanord, C., Spiess, V., Klaus, A., Schwenk, T., and the Expedition 354 Scientists, Bengal Fan. Proceedings of the International Ocean Discovery Program, 354 : College Station, TX (International Ocean Discovery Program). https://doi.org/10.14379/iodp.proc.354.202.2018

2 Department of Geology, Tri-Chandra Campus,Tribhuvan University, Ghantaghar, Kathmandu, Nepal.

${ }^{3}$ Department of Geoscience, Shimane University, Japan. Correspondence author: swostik_adhikari@hotmail.com

${ }^{4}$ Department of Geology, Shinshu University, Japan.

MS 354-202: Received 22 March 2018 • Accepted 30 July 2018 • Published 17 October 2018

This work is distributed under the Creative Commons Attribution 4.0 International (CC BY 4.0) license. (c) BY
} 
ment specimens were also taken from hemipelagic beds. Samples from apparently massive beds containing loose sands that were placed vertically on the core receiving platform to allow the sand to settle to the bottom were not collected for this study.

Sediment samples of approximately $0.1 \mathrm{~g}$ dry weight were placed in glass vials and mixed with $10 \%$ hydrogen peroxide $\left(\mathrm{H}_{2} \mathrm{O}_{2}\right)$. These solutions were held for more than $24 \mathrm{~h}$ to decompose the organic matter contained in the samples. To ensure complete disaggregation of the sediments, an ultrasonic probe was used for approximately 30 $\mathrm{s}$ before analysis of the sample in the laser-analyzer. The grain size analysis was performed by the laser diffraction method using a SALD 3000s (Shimadzu Corp.; range of measureable size: $0.3 \mu \mathrm{m}-3$ $\mathrm{mm})$. A $0.2 \%$ sodium hexametaphosphate $\left[\left(\mathrm{NaPO}_{3}\right)_{6}\right]$ solution was used as a dispersant during the laser analysis. At least five measurements were taken for each sample aliquot to confirm reproducibility, and all results were then averaged to obtain the final value.

\section{Statistical parameters}

Grain size parameters of mean $\left(M_{\mathrm{z}}\right)$, sorting (standard deviation; $\sigma$ ), skewness (SK), and kurtosis $\left(K_{\mathrm{G}}\right)$ as well as sand, silt, and clay weight percentages were calculated for all samples using the phiscale, according to the methods of Folk and Ward (1957). The following mathematical calculations (Folk and Ward, 1957) were applied in this study:

$$
\begin{gathered}
M_{z}=\left(\phi_{16}+\phi_{50}+\phi_{84}\right) / 3, \\
\sigma=\left[\left(\phi_{84}-\phi_{16}\right) / 4\right]+\left[\left(\phi_{95}-\phi_{5}\right) / 6.6\right], \\
\mathrm{SK}=\left[\left(\phi_{16}+\phi_{84}-2 \phi_{50}\right) / 2\left(\phi_{84}-\phi_{16}\right)\right]+ \\
{\left[\left(\phi_{5}+\phi_{95}-2 \phi_{50}\right) / 2\left(\phi_{95}-\phi_{5}\right)\right], \text { and }} \\
K_{\mathrm{G}}=\left(\phi_{95}-\phi_{5}\right) /\left[2.44\left(\phi_{75}-\phi_{25}\right)\right] .
\end{gathered}
$$

Graphical mean, standard deviation, skewness, and kurtosis were plotted against depth to examine the variation of these parameters downhole. Relative proportions of sand, silt, and clay were plotted on the ternary diagram of Shepard (1954) to evaluate the textural characteristics of the sediments. Frequency distribution curves were drawn from the percentages in each grain size to obtain the grain size distribution patterns. Frequency curves of the differing horizons in individual turbidite beds were compared to understand the variations in grain size distribution within them.

\section{Results}

\section{Graphical grain size parameters \\ Holes U1450A and U1450B}

The mean $\left(M_{\mathrm{z}}\right)$ grain size values in these holes range from 2.53 to 8.28 , with an overall average value of 5.36 (Figure F1A; Tables T1, T2; expanded versions of these tables are available in GRAINSIZE in Supplementary material). The mean values indicate that silt-sized particles are the dominant textural class. Most of the mud samples are fine- to medium-grained silts with only a few being coarse-grained silts. In the lower sandy part of the turbidites, the bed base samples range from medium-grained silts to very fine grained sands, whereas the sandy bed middle samples are slightly coarser, ranging from medium-grained silts to fine grained sands. The bed top samples are fine-grained silts to very fine grained sands. The overall upward change in mean grain size values in the sandy parts of the turbidites indicates a slight coarsening-upward trend, which includes some small-scale coarsening-upward trends (e.g., 180 to $50 \mathrm{~m}$ ) (Figure F1A). Standard deviation $(\sigma)$ values range from 0.95 to $2.27 \phi$, with an average of $1.58 \phi$. Most of the samples are poorly sorted, with only a few samples being moderately sorted or very poorly sorted (Figure F1B). Skewness (SK) values range from -0.06 to 0.49 , with an average of 0.15 . Most of the samples fall in the symmetrical and positively skewed category, but seven samples fall in the very positively skewed category (Figure F1C). Kurtosis $\left(K_{\mathrm{G}}\right)$ values range from 0.76 to 2.05 , with an average of 1.09 . Most samples have mesokurtic and leptokurtic distributions, although five samples show very leptokurtic distributions (Figure F1D). The $\sigma$, SK, and $K_{\mathrm{G}}$ values show no significant or systematic variation with depth.

\section{Holes U1451A and U1451B}

Mean grain size values in Site U1451 samples range from 1.69 to $8.02 \phi$ with an overall average value of $5.63 \phi$ (Figure F2A; Tables T3, T4; expanded versions of these tables are available in Supplementary material). The mean $\left(M_{\mathrm{z}}\right)$ values show that silt-size particles are again the major textural class with only a few samples being dominated by sand-size particles. Nearly all of the mud samples are fine- to medium-grained silts, with only three samples classified as coarse-grained silts. In the lower sandy parts, the bed base samples are medium-grained silts to very fine grained sands, whereas the bed middle samples are fine-grained silts to fine-grained sands. The top samples of the sandy beds are fine-grained silts to fine-grained sands, except for a single medium-grained sand near the top of Hole U1451A. The upward change in mean grain size values show smallscale fining (e.g., 250 to $125 \mathrm{~m}$ ) (Figure F2A) as well as coarsening (e.g., 450 to $300 \mathrm{~m}$ ) (Figure F2A) upward trends, whereas the mean grain size values of samples in the sandy parts of the turbidites show a slight coarsening-upward trend. Standard deviation $(\sigma)$ values show almost all samples are poorly sorted except for two that are moderately sorted and one that is well sorted (Figure F2B). The $\sigma$ values range from 0.38 to $2.29 \phi$, with an average value of $1.58 \phi$. Skewness values range from -0.12 to +0.45 with an average of 0.17 . Most of the samples are distributed in the symmetrical and positively skewed categories, although 13 samples are classified as very positively skewed (Figure F2C). Kurtosis values range from 0.78 to 1.40 with an average of 1.08 . Most samples show mesokurtic and leptokurtic distributions, and five samples exhibit platykurtic distributions (Figure F2D). As at Site U1450, the $\sigma$, SK, and $K_{\mathrm{G}}$ values show no significant variation with depth.

\section{Textural classification of the sediments}

The ternary sand-silt-clay plot of Shepard (1954) provides a textural classification of the sediments based on grain size. Sediment samples from Sites U1450 and U1451 are plotted separately in Figure F3. Most of the samples from the base, middle, and top horizons of the sandy parts of the turbidites cluster near the edge between the sand and silt apexes, and the samples from upper muddy horizons cluster near the edge between silt and clay. The figures show that the sediments in both holes are mostly sandy silts, silty sands, and clayey silts with subordinate silts and sands.

\section{Frequency curves}

Frequency curves of the differing horizons of individual turbidites were compared to examine the variation in grain size distribution within these beds. Nineteen turbidite beds from Site U1450 and 40 turbidite beds from Site U1451 were examined in this way. Frequency distribution curves show that three types of beds are present: normally graded, inversely graded, and homogeneous 
Figure F1. Depth variation of textural components in Site U1450 sediments. (A) Mean grain size, (B) standard deviation (sorting), (C) skewness, and (D) kurtosis. Ages after France-Lanord et al. (2016b; see the Site U1450 chapter). Calculation methods after Folk and Ward (1957). f. $=$ fine, v.f. $=$ very fine, C. $=$ coarse, v.c. $=$ very coarse, $\mathrm{m} .=$ medium.

U1450A

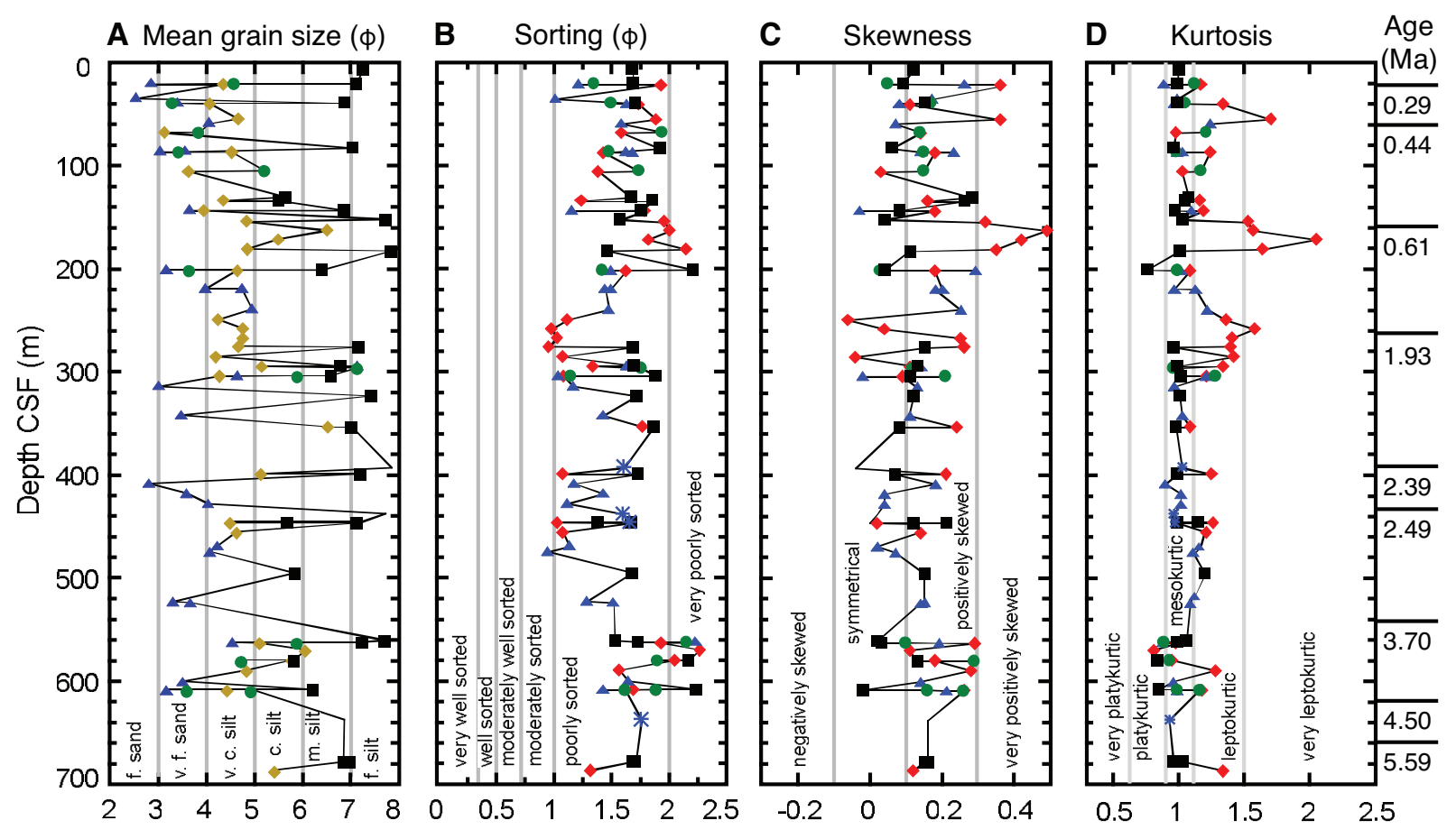

U1450B

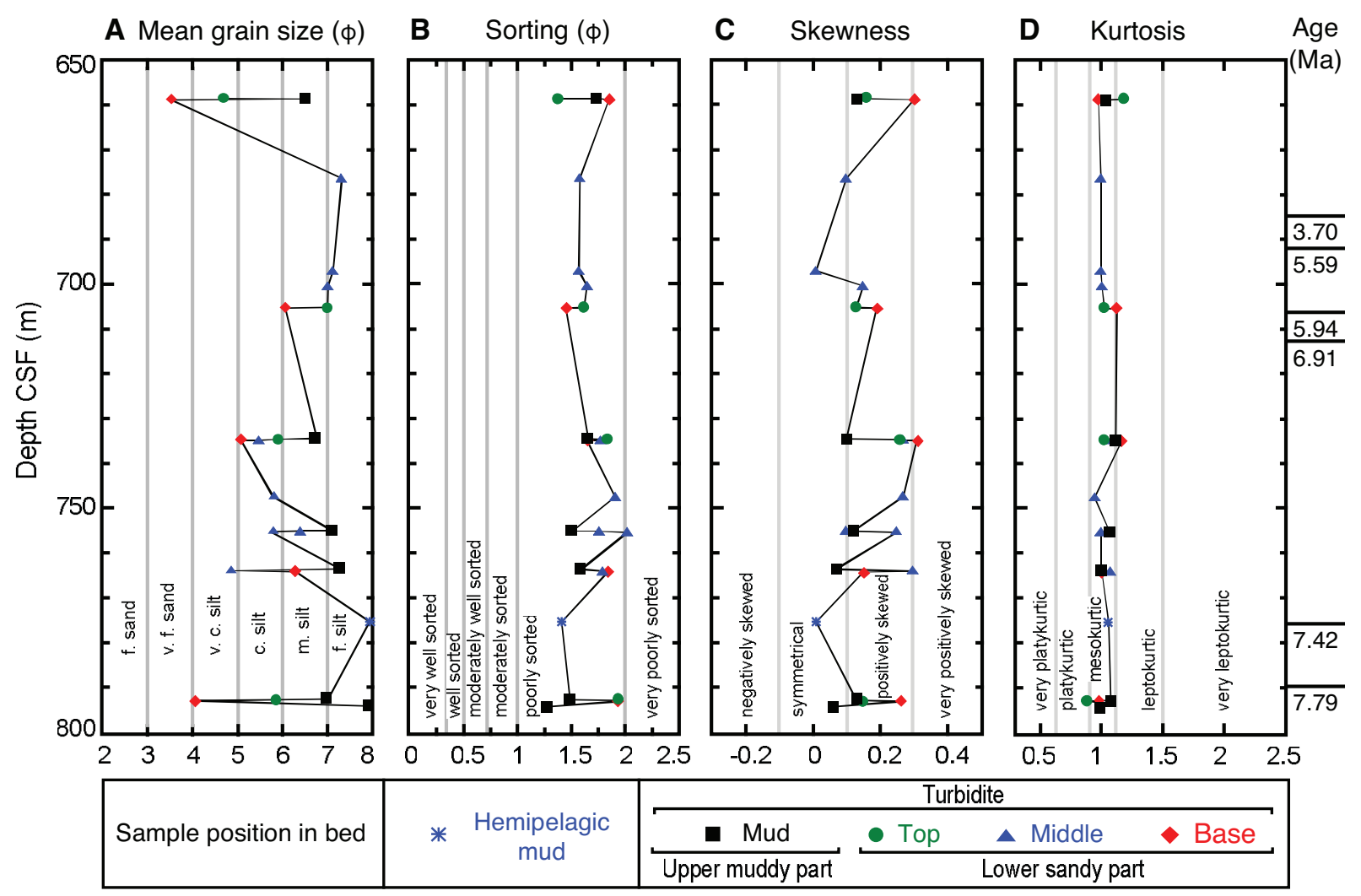


Table T1. Grain size analysis data, Hole U1450A. $M_{z}=$ mean, $\sigma=$ sorting (standard deviation), $S K=$ skewness, $K_{G}=$ kurtosis. Bed position: $F=$ fine (upper muddy part of turbidite); $\mathrm{T}=$ top, $\mathrm{M}=$ middle, $\mathrm{B}=$ base (lower sandy part of turbidite). $\mathrm{Tr}=$ turbidite, $\mathrm{He}=$ hemipelagic mud. Color blocks = individual turbidite sequences and the differing horizons in them. An expanded version of this table is available in GRAINSIZE in Supplementary material. (Continued on next page.) Download table in CSV format.

\begin{tabular}{|c|c|c|c|c|c|c|c|c|c|c|}
\hline $\begin{array}{c}\text { Serial } \\
\text { number }\end{array}$ & $\begin{array}{l}\text { Core, section, } \\
\text { interval }(\mathrm{m})\end{array}$ & $\begin{array}{l}\text { Sand } \\
\text { (wt\%) }\end{array}$ & $\begin{array}{c}\text { Silt } \\
\text { (wt\%) }\end{array}$ & $\begin{array}{l}\text { Clay } \\
\text { (wt\%) }\end{array}$ & $M_{\mathrm{Z}}$ & $\sigma$ & SK & $K_{\mathrm{G}}$ & $\begin{array}{c}\text { Bed } \\
\text { position }\end{array}$ & $\begin{array}{l}\mathrm{Tr} / \\
\mathrm{He}\end{array}$ \\
\hline \multicolumn{11}{|c|}{ 354-U1450A- } \\
\hline 1 & $1 \mathrm{H}-5,6.64-6.69$ & 1.44 & 69.89 & 28.66 & 7.26 & 1.68 & 0.12 & 1.00 & $\mathrm{~F}$ & $\operatorname{Tr}$ \\
\hline 2 & $4 F-1,20.22-20.23$ & 1.64 & 71.74 & 26.62 & 7.11 & 1.69 & 0.09 & 0.99 & $\mathrm{~F}$ & $\operatorname{Tr}$ \\
\hline 3 & 4F-1, 20.31-20.32 & 37.64 & 59.84 & 2.53 & 4.58 & 1.35 & 0.05 & 1.12 & $\mathrm{~T}$ & $\operatorname{Tr}$ \\
\hline 4 & $4 F-1,20.50-20.51$ & 83.99 & 14.83 & 1.04 & 2.85 & 1.22 & 0.26 & 0.89 & M & $\operatorname{Tr}$ \\
\hline 5 & $4 F-1,21.15-21.16$ & 55.86 & 37.61 & 6.53 & 4.35 & 1.93 & 0.36 & 1.17 & B & $\operatorname{Tr}$ \\
\hline 6 & 7F-1, 34.77-34.82 & 92.77 & 6.90 & 0.33 & 2.53 & 1.02 & 0.17 & 0.99 & $M$ & $\operatorname{Tr}$ \\
\hline 7 & $8 F-1,39.12-39.13$ & 2.83 & 74.39 & 22.77 & 6.86 & 1.71 & 0.15 & 0.99 & $\mathrm{~F}$ & $\operatorname{Tr}$ \\
\hline 8 & $8 F-1,39.18-39.19$ & 73.72 & 24.69 & 1.36 & 3.30 & 1.50 & 0.17 & 1.05 & $\mathrm{~T}$ & $\operatorname{Tr}$ \\
\hline 9 & $8 F-1,39.50-39.51$ & 67.95 & 29.85 & 1.65 & 3.39 & 1.64 & 0.08 & 0.97 & $M$ & $\operatorname{Tr}$ \\
\hline 10 & $8 F-1,39.84-39.85$ & 52.73 & 42.36 & 4.91 & 4.06 & 1.74 & 0.11 & 1.34 & B & $\operatorname{Tr}$ \\
\hline 11 & $11 \mathrm{~F}-1,54.53-54.54$ & 41.37 & 50.96 & 7.67 & 4.65 & 1.89 & 0.36 & 1.70 & B & $\operatorname{Tr}$ \\
\hline 12 & $12 \mathrm{~F}-1,58.69-58.70$ & 53.65 & 42.43 & 3.92 & 4.04 & 1.59 & 0.07 & 1.24 & $M$ & $\operatorname{Tr}$ \\
\hline 13 & $14 \mathrm{~F}-1,67.42-67.43$ & 57.96 & 36.78 & 5.26 & 3.84 & 1.94 & 0.14 & 1.21 & $\mathrm{~T}$ & $\operatorname{Tr}$ \\
\hline 14 & $14 \mathrm{~F}-1,67.73-67.74$ & 74.37 & 23.32 & 1.38 & 3.12 & 1.59 & 0.14 & 0.98 & B & $\operatorname{Tr}$ \\
\hline 15 & $17 F-1,82.66-82.72$ & 5.67 & 66.42 & 27.91 & 7.03 & 1.92 & 0.06 & 0.96 & $\mathrm{~F}$ & $\operatorname{Tr}$ \\
\hline 16 & $18 \mathrm{~F}-1,86.32-86.33$ & 70.35 & 28.30 & 1.12 & 3.43 & 1.48 & 0.15 & 0.98 & $\mathrm{~T}$ & $\operatorname{Tr}$ \\
\hline 17 & $18 \mathrm{~F}-1,86.36-86.37$ & 66.49 & 31.18 & 1.91 & 3.55 & 1.63 & 0.14 & 1.03 & $M$ & $\operatorname{Tr}$ \\
\hline 18 & $18 \mathrm{~F}-1,86.60-86.61$ & 74.62 & 22.92 & 1.53 & 3.04 & 1.69 & 0.23 & 0.99 & M & $\operatorname{Tr}$ \\
\hline 19 & $18 \mathrm{~F}-1,86.80-86.81$ & 41.60 & 54.95 & 3.45 & 4.52 & 1.43 & 0.18 & 1.24 & B & $\operatorname{Tr}$ \\
\hline 20 & $22 \mathrm{H}-1,104.93-104.97$ & 26.10 & 66.15 & 7.75 & 5.21 & 1.74 & 0.15 & 1.17 & $\mathrm{~T}$ & $\operatorname{Tr}$ \\
\hline 21 & $22 \mathrm{H}-1,105.46-105.47$ & 65.65 & 32.78 & 1.58 & 3.62 & 1.39 & 0.03 & 1.03 & B & $\operatorname{Tr}$ \\
\hline 22 & $27 F-2,130.27-130.28$ & 17.18 & 72.93 & 9.90 & 5.63 & 1.67 & 0.28 & 1.08 & $\mathrm{~F}$ & $\operatorname{Tr}$ \\
\hline 23 & $28 \mathrm{~F}-1,133.52-133.53$ & 47.46 & 50.69 & 1.85 & 4.34 & 1.24 & 0.16 & 1.16 & B & $\operatorname{Tr}$ \\
\hline 24 & $28 \mathrm{~F}-1,133.74-133.79$ & 23.86 & 65.38 & 10.77 & 5.50 & 1.86 & 0.26 & 1.05 & $\mathrm{~F}$ & $\operatorname{Tr}$ \\
\hline 25 & $30 F-1,143.07-143.08$ & 3.40 & 73.06 & 23.55 & 6.87 & 1.76 & 0.08 & 0.97 & $\mathrm{~F}$ & $\operatorname{Tr}$ \\
\hline 26 & $30 F-1,143.43-143.44$ & 69.40 & 30.37 & 0.04 & 3.65 & 1.16 & -0.03 & 1.10 & M & $\operatorname{Tr}$ \\
\hline 27 & $30 F-1,43.72-143.73$ & 57.18 & 37.87 & 4.95 & 3.94 & 1.79 & 0.18 & 1.19 & B & $\operatorname{Tr}$ \\
\hline 28 & $32 F-1,152.10-152.15$ & 0.35 & 61.51 & 38.14 & 7.72 & 1.58 & 0.04 & 1.03 & $\mathrm{~T}$ & $\operatorname{Tr}$ \\
\hline 29 & $32 F-2,154.17-154.18$ & 36.89 & 54.45 & 8.66 & 4.83 & 1.96 & 0.32 & 1.53 & B & $\operatorname{Tr}$ \\
\hline 30 & $34 \mathrm{~F}-1,162.47-162.48$ & 4.34 & 77.88 & 17.77 & 6.50 & 2.01 & 0.49 & 1.57 & B & $\operatorname{Tr}$ \\
\hline 31 & $36 \mathrm{~F}-1,171.67-171.68$ & 13.68 & 75.46 & 10.86 & 5.48 & 1.82 & 0.42 & 2.05 & B & $\operatorname{Tr}$ \\
\hline 32 & $38 F-1,180.83-180.84$ & 38.17 & 52.63 & 9.20 & 4.84 & 2.15 & 0.35 & 1.64 & B & $\operatorname{Tr}$ \\
\hline 33 & $38 F-2,182.93-182.98$ & 0.26 & 60.81 & 38.94 & 7.84 & 1.47 & 0.11 & 1.01 & $\mathrm{~F}$ & $\operatorname{Tr}$ \\
\hline 34 & $42 F-2,201.19-201.20$ & 18.85 & 55.99 & 25.17 & 6.41 & 2.21 & 0.04 & 0.76 & $\mathrm{~F}$ & $\operatorname{Tr}$ \\
\hline 35 & $42 F-2,201.37-201.38$ & 64.15 & 34.43 & 1.01 & 3.65 & 1.42 & 0.03 & 0.99 & $\mathrm{~T}$ & $\operatorname{Tr}$ \\
\hline 36 & $42 F-2,201.48-201.53$ & 75.93 & 22.88 & 1.19 & 3.17 & 1.50 & 0.29 & 1.02 & M & $\operatorname{Tr}$ \\
\hline 37 & $42-2,201.60-201.61$ & 40.68 & 54.92 & 4.40 & 4.63 & 1.63 & 0.18 & 1.09 & B & $\operatorname{Tr}$ \\
\hline 38 & $46 F 1,219.42-219.47$ & 58.92 & 39.10 & 1.99 & 3.97 & 1.50 & 0.20 & 0.97 & $\mathrm{He}$ & $\mathrm{He}$ \\
\hline 39 & $46 F-1,219.63-219.64$ & 35.33 & 60.69 & 3.98 & 4.73 & 1.45 & 0.18 & 1.13 & $\mathrm{He}$ & $\mathrm{He}$ \\
\hline 40 & 50F-2, 239.31-239.37 & 30.79 & 64.17 & 5.05 & 4.94 & 1.48 & 0.25 & 1.22 & M & $\operatorname{Tr}$ \\
\hline 41 & $52 F-3,249.30-249.31$ & 46.88 & 52.94 & 0.17 & 4.24 & 1.12 & -0.06 & 1.36 & B & $\operatorname{Tr}$ \\
\hline 42 & $54 F-2,258.03-258.04$ & 21.20 & 75.99 & 2.81 & 4.75 & 0.98 & 0.04 & 1.58 & B & $\operatorname{Tr}$ \\
\hline 43 & $56 F-1,266.87-266.88$ & 24.97 & 71.03 & 3.99 & 4.75 & 1.03 & 0.25 & 1.41 & B & $\operatorname{Tr}$ \\
\hline 44 & $58 F-1,275.61-275.62$ & 28.32 & 67.87 & 3.81 & 4.66 & 0.96 & 0.26 & 1.40 & B & $\operatorname{Tr}$ \\
\hline 45 & $58 F-2,276.43-276.49$ & 1.25 & 71.14 & 27.60 & 7.17 & 1.69 & 0.15 & 0.96 & $\mathrm{~F}$ & $\operatorname{Tr}$ \\
\hline 46 & $60 F-1,285.28-285.29$ & 50.13 & 49.67 & 0.20 & 4.19 & 1.08 & -0.04 & 1.42 & B & $\operatorname{Tr}$ \\
\hline 47 & $62 F-1,294.42-294.43$ & 3.04 & 75.34 & 21.62 & 6.79 & 1.70 & 0.13 & 0.99 & $\mathrm{~F}$ & $\operatorname{Tr}$ \\
\hline 48 & $62 F-1,294.66-294.67$ & 1.33 & 72.62 & 26.05 & 7.12 & 1.64 & 0.14 & 1.00 & M & $\operatorname{Tr}$ \\
\hline 49 & $62 F-1,294.72-294.73$ & 20.06 & 75.54 & 4.40 & 5.14 & 1.34 & 0.11 & 1.34 & B & $\operatorname{Tr}$ \\
\hline 50 & 62F-2, 296.27-296.32 & 2.05 & 70.04 & 27.91 & 7.13 & 1.76 & 0.12 & 0.96 & $\mathrm{~T}$ & $\operatorname{Tr}$ \\
\hline 51 & 64F-1, 303.93-303.94 & 8.72 & 70.89 & 20.38 & 6.58 & 1.88 & 0.11 & 1.02 & $\mathrm{~F}$ & $\operatorname{Tr}$ \\
\hline 52 & $64 F-1,303.99-304.00$ & 4.42 & 89.72 & 5.86 & 5.89 & 1.15 & 0.21 & 1.28 & $\mathrm{~T}$ & $\operatorname{Tr}$ \\
\hline 53 & $64 F-1,304.12-304.13$ & 29.52 & 69.72 & 0.76 & 4.63 & 1.04 & -0.02 & 1.21 & M & $\operatorname{Tr}$ \\
\hline 54 & $64 F-1,304.22-304.23$ & 48.47 & 50.06 & 1.48 & 4.27 & 1.09 & 0.09 & 1.21 & B & $\operatorname{Tr}$ \\
\hline 55 & $66 F-1,314.25-314.26$ & 83.79 & 15.63 & 0.58 & 3.00 & 1.17 & 0.13 & 0.97 & M & $\operatorname{Tr}$ \\
\hline 56 & $68 F-1,323.61-323.66$ & 1.17 & 67.64 & 31.19 & 7.42 & 1.72 & 0.12 & 1.01 & $\mathrm{~F}$ & $\operatorname{Tr}$ \\
\hline 57 & $72 F-1,342.35-342.36$ & 69.15 & 29.14 & 1.71 & 3.47 & 1.43 & 0.11 & 1.03 & M & $\operatorname{Tr}$ \\
\hline 58 & 74F-2, 353.38-353.40 & 5.68 & 76.04 & 18.28 & 6.51 & 1.77 & 0.24 & 1.09 & B & $\operatorname{Tr}$ \\
\hline 59 & $74 F-2,353.40-353.42$ & 5.03 & 67.98 & 26.99 & 7.01 & 1.87 & 0.08 & 0.98 & $\mathrm{~F}$ & $\operatorname{Tr}$ \\
\hline 60 & $82 F-3,392.88-392.93$ & 0.19 & 57.10 & 42.71 & 7.84 & 1.61 & -0.04 & 1.03 & $\mathrm{He}$ & $\mathrm{He}$ \\
\hline 61 & 84F-1, 399.19-399.20 & 16.24 & 80.89 & 2.87 & 5.12 & 1.08 & 0.21 & 1.25 & $\mathrm{~B}$ & $\operatorname{Tr}$ \\
\hline 62 & 84F-1, 399.21-399.27 & 2.29 & 68.79 & 28.92 & 7.19 & 1.73 & 0.07 & 0.99 & $\mathrm{~F}$ & $\operatorname{Tr}$ \\
\hline 63 & $86 F-1,408.72-408.73$ & 85.94 & 13.60 & 0.46 & 2.80 & 1.18 & 0.18 & 0.90 & M & $\operatorname{Tr}$ \\
\hline 64 & $88 F-1,418.50-418.51$ & 66.05 & 32.74 & 1.21 & 3.58 & 1.43 & 0.04 & 1.02 & M & $\operatorname{Tr}$ \\
\hline 65 & $90 F-1,428.12-428.13$ & 55.67 & 43.88 & 0.44 & 4.03 & 1.12 & 0.04 & 1.02 & M & $\operatorname{Tr}$ \\
\hline
\end{tabular}


Table T1 (continued).

\begin{tabular}{|c|c|c|c|c|c|c|c|c|c|c|}
\hline $\begin{array}{c}\text { Serial } \\
\text { number }\end{array}$ & $\begin{array}{l}\text { Core, section, } \\
\text { interval (m) }\end{array}$ & $\begin{array}{l}\text { Sand } \\
\text { (wt\%) }\end{array}$ & $\begin{array}{c}\text { Silt } \\
\text { (wt\%) }\end{array}$ & $\begin{array}{l}\text { Clay } \\
\text { (wt\%) }\end{array}$ & $M_{\mathrm{z}}$ & $\sigma$ & SK & $K_{\mathrm{G}}$ & $\begin{array}{l}\text { Bed } \\
\text { position }\end{array}$ & $\begin{array}{l}\mathrm{Tr} / \\
\mathrm{He}\end{array}$ \\
\hline 66 & $92 \mathrm{~F}-2,438.21-438.22$ & 0.41 & 60.03 & 39.56 & 7.72 & 1.60 & 0.02 & 0.96 & $\mathrm{He}$ & $\mathrm{He}$ \\
\hline 67 & $94 \mathrm{~F}-1,446.28-446.29$ & 1.76 & 69.01 & 29.22 & 7.20 & 1.66 & -0.00 & 0.97 & $\mathrm{He}$ & $\mathrm{He}$ \\
\hline 68 & $94 \mathrm{~F}-1,446.39-446.40$ & 11.04 & 82.12 & 6.84 & 5.68 & 1.39 & 0.21 & 1.15 & $\mathrm{~F}$ & $\operatorname{Tr}$ \\
\hline 69 & $94 F-1,446.49-446.50$ & 33.84 & 65.25 & 0.91 & 4.48 & 1.03 & 0.02 & 1.26 & B & $\operatorname{Tr}$ \\
\hline 70 & $94 \mathrm{~F}-1,446.70-446.75$ & 1.28 & 72.27 & 26.45 & 7.12 & 1.67 & 0.12 & 0.99 & $\mathrm{~F}$ & $\operatorname{Tr}$ \\
\hline 71 & $96 \mathrm{~F}-1,456.07-456.08$ & 32.42 & 65.21 & 2.37 & 4.62 & 1.08 & 0.14 & 1.21 & B & $\operatorname{Tr}$ \\
\hline 72 & $98 \mathrm{~F}-3,469.45-469.46$ & 47.26 & 52.16 & 0.58 & 4.22 & 1.14 & 0.02 & 1.01 & M & $\operatorname{Tr}$ \\
\hline 73 & 100F-1, 475.21-475.22 & 57.06 & 42.85 & 0.09 & 4.07 & 0.95 & 0.07 & 1.11 & M & $\operatorname{Tr}$ \\
\hline 74 & $104 F-2,495.65-495.70$ & 13.31 & 76.70 & 9.98 & 5.83 & 1.68 & 0.15 & 1.20 & $\mathrm{~F}$ & $\operatorname{Tr}$ \\
\hline 75 & $110 F-1,522.99-523.04$ & 75.53 & 23.20 & 1.25 & 3.30 & 1.29 & 0.15 & 1.00 & M & $\operatorname{Tr}$ \\
\hline 76 & $110 F-3,524.49-524.50$ & 65.29 & 32.14 & 2.57 & 3.67 & 1.52 & 0.14 & 1.09 & M & $\operatorname{Tr}$ \\
\hline 77 & $119 F-1,561.03-561.09$ & 0.55 & 61.51 & 37.94 & 7.71 & 1.53 & 0.02 & 1.06 & $\mathrm{~F}$ & $\operatorname{Tr}$ \\
\hline 78 & $120 X-2,562.42-562.43$ & 2.48 & 67.76 & 29.76 & 7.22 & 1.73 & 0.03 & 0.99 & $\mathrm{~F}$ & $\operatorname{Tr}$ \\
\hline 79 & $120 X-2,562.52-562.53$ & 23.71 & 60.33 & 15.96 & 5.88 & 2.15 & 0.10 & 0.89 & $\mathrm{~T}$ & $\operatorname{Tr}$ \\
\hline 80 & $120 \mathrm{X}-2,562.87-562.88$ & 47.18 & 44.50 & 7.07 & 4.53 & 2.23 & 0.19 & 0.88 & M & $\operatorname{Tr}$ \\
\hline 81 & $120 X-2,563.25-563.26$ & 36.05 & 54.64 & 9.30 & 5.10 & 1.93 & 0.29 & 0.98 & B & $\operatorname{Tr}$ \\
\hline 82 & $121 \mathrm{X}-\mathrm{CC}, 570.42-570.43$ & 25.75 & 54.34 & 19.91 & 6.05 & 2.27 & 0.11 & 0.81 & B & $\operatorname{Tr}$ \\
\hline 83 & $122 \mathrm{X}-1,580.10-580.11$ & 26.61 & 57.35 & 16.04 & 5.82 & 2.17 & 0.13 & 0.84 & $\mathrm{~F}$ & $\operatorname{Tr}$ \\
\hline 84 & $122 \mathrm{X}-1,580.18-580.19$ & 44.76 & 48.51 & 6.73 & 4.74 & 1.90 & 0.29 & 0.93 & T & $\operatorname{Tr}$ \\
\hline 85 & $122 X-1,580.37-580.38$ & 24.11 & 62.14 & 13.75 & 5.77 & 2.05 & 0.18 & 0.95 & B & $\operatorname{Tr}$ \\
\hline 86 & $123 X-1,589.87-589.88$ & 34.71 & 59.67 & 5.63 & 4.83 & 1.57 & 0.28 & 1.28 & B & $\operatorname{Tr}$ \\
\hline 87 & $124 F-2,600.93-600.94$ & 65.25 & 32.15 & 2.60 & 3.50 & 1.65 & 0.14 & 0.96 & M & $\operatorname{Tr}$ \\
\hline 88 & $126 \mathrm{~F}-1,608.55-608.56$ & 20.61 & 58.28 & 21.11 & 6.22 & 2.24 & -0.02 & 0.85 & $\mathrm{~F}$ & $\operatorname{Tr}$ \\
\hline 89 & $126 \mathrm{~F}-1,608.63-608.64$ & 62.91 & 33.58 & 2.84 & 3.60 & 1.89 & 0.16 & 0.99 & $\mathrm{~T}$ & $\operatorname{Tr}$ \\
\hline 90 & $126 \mathrm{~F}-1,608.85-608.86$ & 75.93 & 23.00 & 0.50 & 3.17 & 1.43 & 0.21 & 0.99 & M & $\operatorname{Tr}$ \\
\hline 91 & $126 \mathrm{~F}-1,609.10-609.11$ & 48.32 & 46.39 & 5.29 & 4.42 & 1.69 & 0.26 & 1.18 & B & $\operatorname{Tr}$ \\
\hline 92 & $126 \mathrm{~F}-1,609.16-609.20$ & 33.14 & 60.62 & 6.24 & 4.93 & 1.62 & 0.26 & 1.16 & $\mathrm{~T}$ & $\operatorname{Tr}$ \\
\hline 93 & $132 X-1,638.34-638.38$ & 1.95 & 74.37 & 23.68 & 6.86 & 1.76 & 0.16 & 0.93 & $\mathrm{He}$ & $\mathrm{He}$ \\
\hline 94 & $136 X-2,678.39-678.43$ & 2.34 & 75.11 & 22.55 & 6.84 & 1.71 & 0.16 & 0.96 & $\mathrm{~F}$ & $\mathrm{Tr}$ \\
\hline 95 & $136 \mathrm{X}-2,678.47-678.48$ & 2.32 & 73.57 & 24.11 & 6.99 & 1.69 & 0.15 & 1.03 & $\mathrm{~F}$ & $\operatorname{Tr}$ \\
\hline 96 & $137 X-1,687.24-687.25$ & 14.45 & 80.74 & 4.81 & 5.40 & 1.32 & 0.12 & 1.34 & B & $\mathrm{Tr}$ \\
\hline
\end{tabular}

Table T2. Grain size analysis data, Hole U1450B. $M_{\mathrm{z}}=$ mean, $\sigma=$ sorting (standard deviation), $\mathrm{SK}=$ skewness, $K_{\mathrm{G}}=$ kurtosis. Bed position: $\mathrm{T}=$ top, $\mathrm{M}=$ middle, $\mathrm{B}=$ base (lower sandy part of turbidite). $\mathrm{Tr}=$ turbidite, $\mathrm{He}=$ hemipelagic mud. Color blocks = individual turbidite sequences and the differing horizons within them. An expanded version of this table is available in GRAINSIZE in Supplementary material. Download table in CSV format.

\begin{tabular}{|c|c|c|c|c|c|c|c|c|c|c|}
\hline $\begin{array}{c}\text { Serial } \\
\text { number }\end{array}$ & $\begin{array}{l}\text { Core, section, } \\
\text { interval }(\mathrm{m})\end{array}$ & $\begin{array}{l}\text { Sand } \\
\text { (wt\%) }\end{array}$ & $\begin{array}{c}\text { Silt } \\
\text { (wt\%) }\end{array}$ & $\begin{array}{l}\text { Clay } \\
\text { (wt } \%)\end{array}$ & $M_{\mathrm{z}}$ & $\sigma$ & SK & $K_{\mathrm{G}}$ & $\begin{array}{c}\text { Bed } \\
\text { position }\end{array}$ & $\begin{array}{l}\mathrm{Tr} / \\
\mathrm{He}\end{array}$ \\
\hline \multicolumn{11}{|c|}{ 354-U1450B- } \\
\hline 1 & $7 R-2,658.51-658.52$ & 36.26 & 60.23 & 3.51 & 4.67 & 1.39 & 0.16 & 1.18 & T & $\operatorname{Tr}$ \\
\hline 2 & 7R-2, 658.65-658.66 & 7.22 & 75.13 & 17.65 & 6.50 & 1.75 & 0.13 & 1.03 & $\mathrm{~F}$ & $\operatorname{Tr}$ \\
\hline 3 & $7 R-2,658.84-658.85$ & 66.84 & 29.96 & 2.67 & 3.50 & 1.86 & 0.30 & 0.97 & B & $\operatorname{Tr}$ \\
\hline 4 & 9R-1, 676.29-676.33 & 0.54 & 69.90 & 29.56 & 7.31 & 1.59 & 0.10 & 0.99 & M & $\operatorname{Tr}$ \\
\hline 5 & $11 R-2,696.92-696.94$ & 1.10 & 72.83 & 26.07 & 7.12 & 1.58 & 0.01 & 0.99 & M & $\operatorname{Tr}$ \\
\hline 6 & $11 \mathrm{R}-5,700.33-700.38$ & 1.74 & 73.89 & 24.37 & 7.01 & 1.66 & 0.15 & 1.00 & M & $\operatorname{Tr}$ \\
\hline 7 & $12 \mathrm{R}-1,705.15-705.16$ & 1.87 & 74.71 & 23.42 & 6.99 & 1.63 & 0.13 & 1.02 & $\mathrm{~T}$ & $\operatorname{Tr}$ \\
\hline 8 & $12 \mathrm{R}-1,705.27-705.28$ & 7.23 & 82.89 & 9.88 & 6.04 & 1.46 & 0.19 & 1.12 & B & $\operatorname{Tr}$ \\
\hline 9 & $15 R-1,734.44-734.45$ & 4.75 & 75.74 & 19.51 & 6.75 & 1.67 & 0.10 & 1.11 & $\mathrm{~F}$ & $\operatorname{Tr}$ \\
\hline 10 & $15 R-1,734.57-734.58$ & 15.76 & 70.91 & 13.33 & 5.88 & 1.85 & 0.26 & 1.02 & $\mathrm{~T}$ & $\operatorname{Tr}$ \\
\hline 11 & 15R-1, 734.71-734.72 & 23.17 & 66.93 & 9.90 & 5.47 & 1.78 & 0.27 & 1.08 & M & $\operatorname{Tr}$ \\
\hline 12 & $15 R-1,734.79-734.80$ & 30.71 & 62.23 & 7.07 & 5.07 & 1.66 & 0.31 & 1.16 & B & $\operatorname{Tr}$ \\
\hline 14 & 17R-2, 754.93-754.94 & 0.93 & 75.33 & 23.74 & 7.11 & 1.52 & 0.12 & 1.06 & $\mathrm{~F}$ & $\operatorname{Tr}$ \\
\hline 15 & 17R-2, 755.01-755.02 & 9.28 & 74.31 & 16.41 & 6.39 & 1.77 & 0.10 & 1.04 & M & $\operatorname{Tr}$ \\
\hline 16 & $17 R-2,755.10-755.12$ & 23.48 & 63.06 & 13.46 & 5.72 & 2.03 & 0.25 & 0.99 & M & $\operatorname{Tr}$ \\
\hline 17 & $18 \mathrm{R}-1,763.50-763.51$ & 0.65 & 70.18 & 29.17 & 7.28 & 1.60 & 0.07 & 0.99 & $\mathrm{~F}$ & $\operatorname{Tr}$ \\
\hline 18 & 18R-1, 763.86-763.87 & 39.57 & 53.20 & 7.23 & 4.86 & 1.80 & 0.30 & 1.07 & M & $\operatorname{Tr}$ \\
\hline 19 & 18R-1, 764.08-764.09 & 11.19 & 72.18 & 16.63 & 6.28 & 1.85 & 0.15 & 1.00 & B & $\operatorname{Tr}$ \\
\hline 20 & $19 R-3,775.18-775.20$ & 0.13 & 56.04 & 43.83 & 7.94 & 1.42 & 0.01 & 1.05 & $\mathrm{He}$ & $\mathrm{He}$ \\
\hline 21 & 21R-1, 792.60-792.61 & 0.78 & 77.61 & 21.61 & 7.01 & 1.50 & 0.13 & 1.07 & $\mathrm{~F}$ & $\operatorname{Tr}$ \\
\hline 22 & 21R-1, 792.69-792.70 & 21.47 & 64.59 & 13.94 & 5.86 & 1.95 & 0.15 & 0.88 & $\mathrm{~T}$ & $\operatorname{Tr}$ \\
\hline 23 & 21R-1, 792.90-792.91 & 56.97 & 37.95 & 4.63 & 4.06 & 1.94 & 0.26 & 0.98 & B & $\operatorname{Tr}$ \\
\hline 24 & 21R-2, 794.09-794.11 & 0.01 & 47.78 & 52.21 & 8.28 & 1.29 & 0.06 & 0.98 & $\mathrm{~F}$ & $\operatorname{Tr}$ \\
\hline
\end{tabular}


Figure F2. Depth variation of textural components in Site U1451 sediments. (A) Mean grain size, (B) standard deviation (sorting), (C) skewness, and (D) kurtosis. Ages after France-Lanord et al. (2016c; see the Site U1451 chapter). f. $=$ fine, v.f. $=$ very fine, $c .=$ coarse, v.c. $=$ very coarse, $\mathrm{m} .=\mathrm{medium}$.

\section{U1451A}
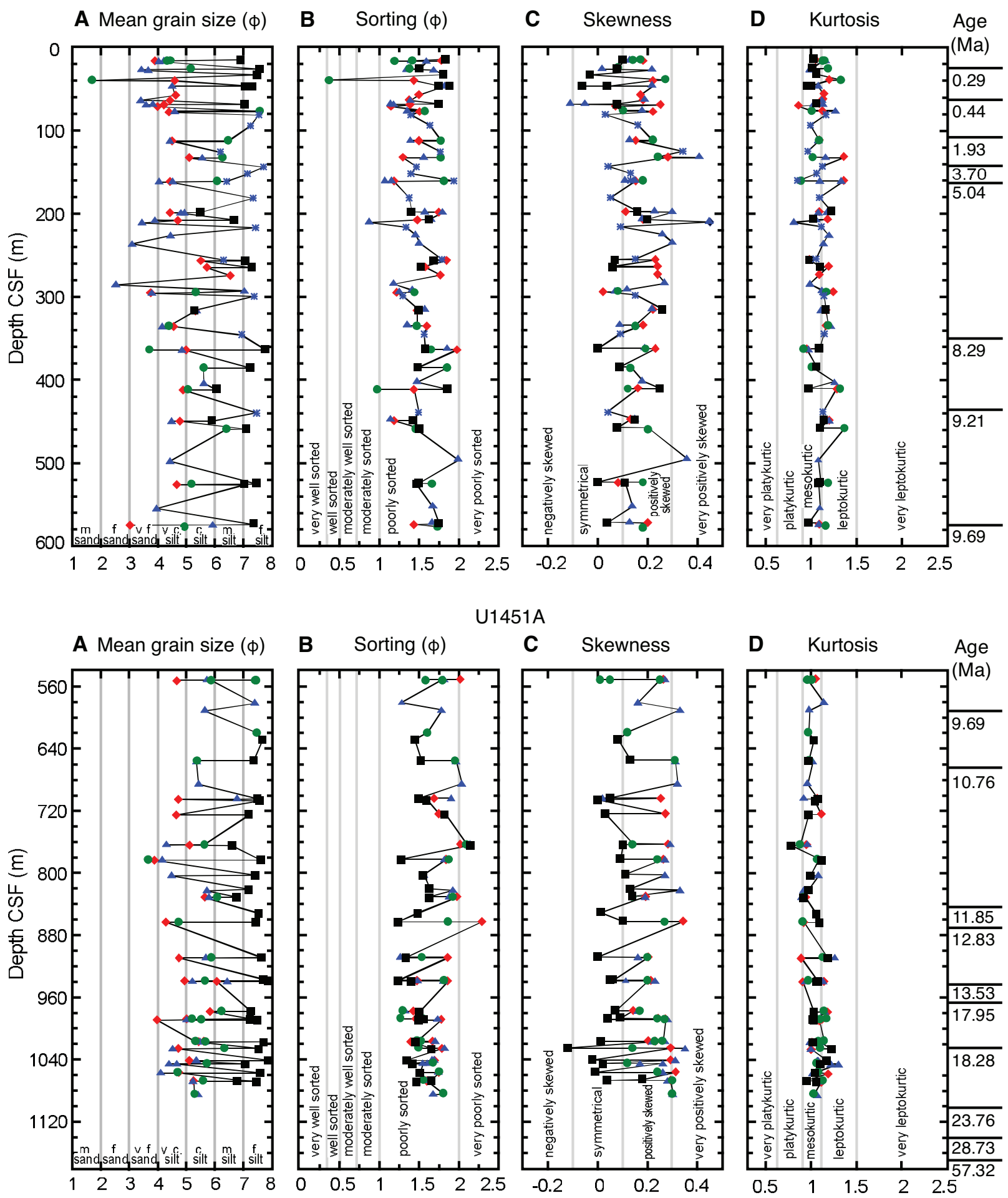

U1451A
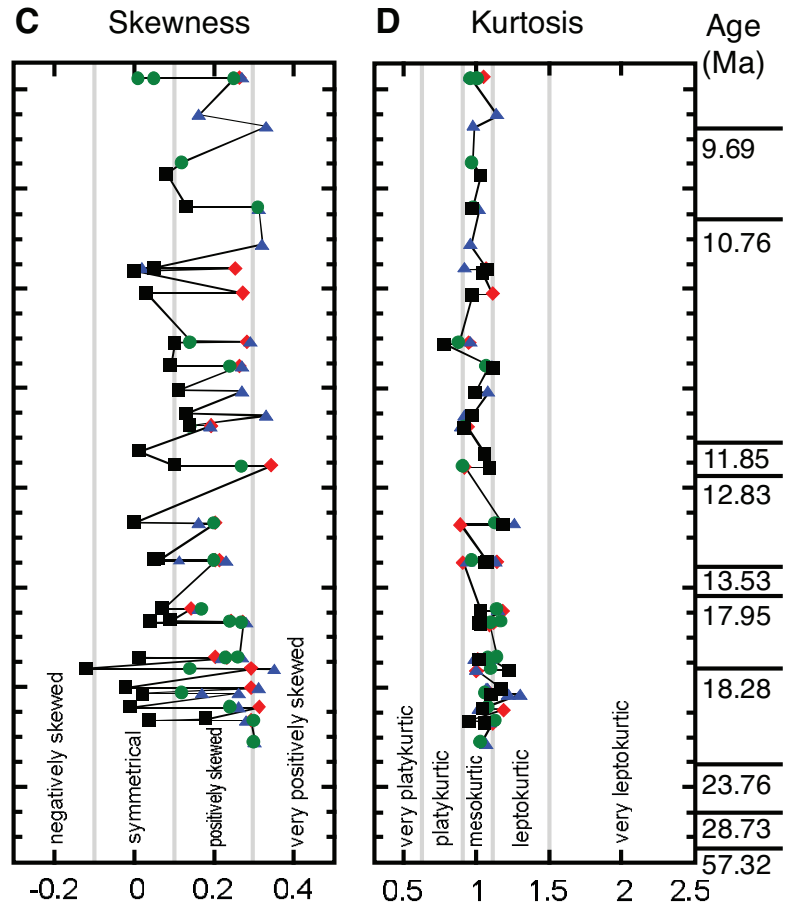
Table T3. Grain size analysis data, Hole U1451A. $M_{z}=$ mean, $\sigma=$ sorting (standard deviation), $S K=$ skewness, $K_{\mathrm{G}}=$ kurtosis. Bed position: $\mathrm{F}=$ fine (upper muddy part of turbidite), $\mathrm{T}=$ top, $\mathrm{M}=$ middle, $\mathrm{B}=$ base, respectively (lower sandy part of turbidite). $\mathrm{Tr}=$ turbidite, $\mathrm{He}=$ hemipelagic mud. Color blocks = individual turbidite sequences and the differing horizons in them. An expanded version of this table is available in GRAINSIZE in Supplementary material. (Continued on next page.) Download table in CSV format.

\begin{tabular}{|c|c|c|c|c|c|c|c|c|c|c|}
\hline $\begin{array}{c}\text { Serial } \\
\text { number }\end{array}$ & $\begin{array}{l}\text { Core, section, } \\
\text { interval }(\mathrm{m})\end{array}$ & $\begin{array}{l}\text { Sand } \\
\text { (wt\%) }\end{array}$ & $\begin{array}{c}\text { Silt } \\
\text { (wt\%) }\end{array}$ & $\begin{array}{l}\text { Clay } \\
\text { (wt\%) }\end{array}$ & $M_{\mathrm{Z}}$ & $\sigma$ & SK & $K_{\mathrm{G}}$ & $\begin{array}{c}\text { Bed } \\
\text { position }\end{array}$ & $\begin{array}{l}\mathrm{Tr} / \\
\mathrm{He}\end{array}$ \\
\hline \multicolumn{11}{|c|}{ 354-U1451A- } \\
\hline 1 & $2 \mathrm{H}-6,14.53-14.58$ & 5.29 & 70.12 & 24.58 & 6.91 & 1.84 & 0.10 & 1.03 & $\mathrm{~F}$ & $\operatorname{Tr}$ \\
\hline 2 & $3 \mathrm{H}-1,16.13-16.14$ & 42.69 & 54.22 & 3.08 & 4.44 & 1.42 & 0.17 & 1.15 & $\mathrm{~T}$ & $\operatorname{Tr}$ \\
\hline 3 & $3 \mathrm{H}-1,16.40-16.41$ & 50.31 & 47.39 & 2.30 & 4.21 & 1.41 & 0.13 & 1.15 & $M$ & $\operatorname{Tr}$ \\
\hline 4 & $3 \mathrm{H}-1,16.48-16.49$ & 55.54 & 37.08 & 3.27 & 3.90 & 1.78 & 0.18 & 1.05 & B & $\operatorname{Tr}$ \\
\hline 5 & $3 \mathrm{H}-1,16.58-16.59$ & 46.59 & 51.47 & 1.94 & 4.32 & 1.20 & 0.14 & 1.13 & $\mathrm{~T}$ & $\operatorname{Tr}$ \\
\hline 6 & $3 \mathrm{H}-1,16.93-16.94$ & 53.94 & 42.99 & 3.07 & 4.04 & 1.60 & 0.10 & 1.12 & $M$ & $\operatorname{Tr}$ \\
\hline 7 & $4 \mathrm{H}-2,25.74-25.75$ & 0.05 & 65.68 & 34.27 & 7.57 & 1.51 & 0.08 & 1.01 & $\mathrm{~F}$ & $\operatorname{Tr}$ \\
\hline 8 & $4 \mathrm{H}-2,25.79-25.80$ & 22.15 & 73.36 & 4.49 & 5.15 & 1.39 & 0.08 & 1.19 & $\mathrm{~T}$ & $\operatorname{Tr}$ \\
\hline 9 & $4 \mathrm{H}-2,26.67-26.68$ & 71.13 & 27.98 & 0.90 & 3.43 & 1.35 & 0.02 & 0.98 & M & $\operatorname{Tr}$ \\
\hline 10 & $4 \mathrm{H}-3,27.56-27.57$ & 64.48 & 33.01 & 2.30 & 3.67 & 1.69 & 0.22 & 0.98 & $M$ & $\mathrm{Tr}$ \\
\hline 11 & $5 \mathrm{H}-3,32.67-32.72$ & 2.70 & 61.68 & 35.63 & 7.49 & 1.81 & -0.03 & 1.06 & $\mathrm{~F}$ & $\operatorname{Tr}$ \\
\hline 12 & 7H-1, 39.76-39.77 & 98.86 & 1.14 & 0.00 & 1.69 & 0.38 & 0.27 & 1.33 & $\mathrm{~T}$ & $\operatorname{Tr}$ \\
\hline 13 & $7 \mathrm{H}-1,40.26-40.27$ & 40.92 & 55.57 & 3.51 & 4.58 & 1.44 & 0.22 & 1.20 & B & $\operatorname{Tr}$ \\
\hline 14 & $8 \mathrm{H}-1,46.70-46.76$ & 1.86 & 66.65 & 31.49 & 7.32 & 1.75 & 0.04 & 1.00 & $\mathrm{~F}$ & $\operatorname{Tr}$ \\
\hline 15 & $8 \mathrm{H}-1,46.79-46.80$ & 5.94 & 64.54 & 29.53 & 7.06 & 1.89 & -0.06 & 0.96 & $\mathrm{~F}$ & $\operatorname{Tr}$ \\
\hline 16 & $8 \mathrm{H}-1,46.85-46.86$ & 47.15 & 47.34 & 5.37 & 4.50 & 1.83 & 0.22 & 1.07 & $M$ & $\operatorname{Tr}$ \\
\hline 17 & $10 F-2,57.61-57.62$ & 39.65 & 56.52 & 3.82 & 4.61 & 1.50 & 0.17 & 1.14 & B & $\operatorname{Tr}$ \\
\hline 18 & $11 \mathrm{~F}-3,63.85-63.90$ & 73.03 & 24.61 & 1.80 & 3.40 & 1.40 & 0.19 & 1.11 & $M$ & $\operatorname{Tr}$ \\
\hline 19 & $11 F-3,63.91-63.92$ & 44.60 & 52.49 & 2.91 & 4.42 & 1.38 & 0.18 & 1.13 & $B$ & $\operatorname{Tr}$ \\
\hline 20 & $12 F-3,68.27-68.28$ & 3.20 & 71.18 & 25.62 & 7.05 & 1.75 & 0.08 & 1.06 & $\mathrm{~F}$ & $\operatorname{Tr}$ \\
\hline 21 & $12 F-3,68.51-68.52$ & 62.06 & 37.93 & 0.00 & 3.84 & 1.15 & -0.11 & 1.12 & M & $\operatorname{Tr}$ \\
\hline 22 & $12 F-3,68.91-68.92$ & 69.71 & 30.23 & 0.06 & 3.59 & 1.19 & -0.05 & 1.02 & $M$ & $\mathrm{Tr}$ \\
\hline 23 & $12 F-4,69.31-69.32$ & 52.92 & 44.07 & 3.01 & 4.22 & 1.39 & 0.25 & 1.09 & B & $\operatorname{Tr}$ \\
\hline 24 & $13 F-2,71.26-71.27$ & 56.78 & 43.20 & 0.01 & 3.99 & 1.15 & 0.07 & 0.86 & B & $\operatorname{Tr}$ \\
\hline 25 & $14 \mathrm{~F}-2,76.92-76.93$ & 0.47 & 65.54 & 33.99 & 7.57 & 1.58 & 0.10 & 1.01 & $\mathrm{~T}$ & $\operatorname{Tr}$ \\
\hline 26 & $14 F-2,77.02-77.03$ & 36.61 & 60.09 & 3.31 & 4.59 & 1.36 & 0.18 & 1.26 & $M$ & $\operatorname{Tr}$ \\
\hline 27 & $14 F-3,77.87-77.88$ & 48.11 & 48.55 & 3.34 & 4.39 & 1.51 & 0.22 & 1.12 & B & $\operatorname{Tr}$ \\
\hline 28 & $15 F-2,81.31-81.35$ & 0.93 & 67.86 & 31.21 & 7.53 & 1.40 & 0.03 & 1.16 & $\mathrm{He}$ & $\mathrm{He}$ \\
\hline 29 & $18 \mathrm{~F}-1,94.14-94.19$ & 0.96 & 71.11 & 27.93 & 7.24 & 1.64 & 0.16 & 0.99 & $\mathrm{He}$ & $\mathrm{He}$ \\
\hline 30 & $22 \mathrm{H}-1,112.52-112.53$ & 6.37 & 76.12 & 17.51 & 6.46 & 1.78 & 0.22 & 1.09 & $\mathrm{~T}$ & $\operatorname{Tr}$ \\
\hline 31 & $22 \mathrm{H}-1,112.61-112.62$ & 44.39 & 52.96 & 2.65 & 4.42 & 1.40 & 0.13 & 1.07 & M & $\operatorname{Tr}$ \\
\hline 32 & $22 \mathrm{H}-1,112.70-112.71$ & 42.78 & 54.04 & 3.18 & 4.50 & 1.50 & 0.15 & 1.09 & B & $\operatorname{Tr}$ \\
\hline 33 & $23 \mathrm{H}-5,125.99-126.04$ & 7.47 & 76.18 & 16.35 & 6.19 & 1.77 & 0.34 & 0.96 & $\mathrm{He}$ & $\mathrm{He}$ \\
\hline 34 & $24 \mathrm{H}-2,132.71-132.72$ & 8.53 & 75.09 & 16.38 & 6.27 & 1.78 & 0.24 & 1.02 & $T$ & $\operatorname{Tr}$ \\
\hline 35 & $24 \mathrm{H}-2,132.79-132.84$ & 15.21 & 75.03 & 9.76 & 5.56 & 1.57 & 0.41 & 1.15 & $M$ & $\operatorname{Tr}$ \\
\hline 36 & $24 \mathrm{H}-2,132.84-132.85$ & 19.66 & 75.69 & 4.65 & 5.09 & 1.30 & 0.28 & 1.36 & B & $\operatorname{Tr}$ \\
\hline 37 & $25 \mathrm{H}-3,144.00-144.05$ & 0.55 & 63.32 & 36.13 & 7.70 & 1.47 & 0.04 & 1.12 & $\mathrm{He}$ & $\mathrm{He}$ \\
\hline 38 & $26 \mathrm{H}-3,152.35-152.39$ & 0.32 & 76.94 & 22.73 & 7.13 & 1.40 & 0.13 & 1.06 & $\mathrm{He}$ & $\mathrm{He}$ \\
\hline 39 & $27 \mathrm{H}-2,161.02-161.03$ & 10.37 & 69.15 & 20.48 & 6.41 & 1.94 & 0.13 & 0.85 & $\mathrm{He}$ & $\mathrm{He}$ \\
\hline 40 & $27 \mathrm{H}-2,161.14-161.15$ & 13.73 & 71.47 & 14.80 & 6.07 & 1.82 & 0.18 & 0.89 & $\mathrm{~T}$ & $\operatorname{Tr}$ \\
\hline 41 & $27 \mathrm{H}-2,161.24-161.25$ & 37.52 & 60.78 & 1.70 & 4.52 & 1.16 & 0.15 & 1.32 & M & $\operatorname{Tr}$ \\
\hline 42 & $27 \mathrm{H}-2,161.26-161.31$ & 57.50 & 42.14 & 0.36 & 4.05 & 1.08 & 0.11 & 1.09 & $M$ & $\operatorname{Tr}$ \\
\hline 43 & $27 \mathrm{H}-2,161.31-161.32$ & 42.99 & 55.77 & 1.25 & 4.42 & 1.19 & 0.15 & 1.36 & B & $\operatorname{Tr}$ \\
\hline 44 & $29 \mathrm{H}-3,181.35-181.40$ & 0.31 & 73.03 & 26.66 & 7.34 & 1.38 & 0.05 & 1.09 & $\mathrm{He}$ & $\mathrm{He}$ \\
\hline 45 & 31F-1, 198.53-198.54 & 14.52 & 79.73 & 5.75 & 5.49 & 1.41 & 0.16 & 1.22 & $\mathrm{~F}$ & $\operatorname{Tr}$ \\
\hline 46 & $31 F-1,198.65-198.66$ & 32.30 & 62.11 & 5.59 & 4.93 & 1.58 & 0.23 & 1.17 & $M$ & $\operatorname{Tr}$ \\
\hline 47 & $31 F-1,198.75-198.76$ & 44.78 & 50.98 & 4.24 & 4.42 & 1.75 & 0.11 & 1.09 & B & $\operatorname{Tr}$ \\
\hline 48 & 31F-2, 199.30-199.35 & 41.55 & 51.62 & 6.83 & 4.80 & 1.80 & 0.30 & 1.07 & M & $\operatorname{Tr}$ \\
\hline 49 & 33F-1, 207.41-207.42 & 2.43 & 78.69 & 18.88 & 6.68 & 1.63 & 0.20 & 1.02 & $\mathrm{~F}$ & $\operatorname{Tr}$ \\
\hline 50 & $33 F-1,207.74-207.75$ & 59.84 & 37.39 & 2.77 & 3.89 & 1.66 & 0.18 & 1.05 & M & $\operatorname{Tr}$ \\
\hline 51 & $33 F-1,208.09-208.10$ & 36.29 & 59.88 & 3.84 & 4.70 & 1.48 & 0.18 & 1.18 & B & $\operatorname{Tr}$ \\
\hline 52 & $33 F-4,210.91-210.96$ & 76.07 & 23.93 & 0.00 & 3.44 & 0.88 & 0.45 & 0.80 & $M$ & $\operatorname{Tr}$ \\
\hline 53 & $35 F-1,216.77-216.82$ & 0.32 & 71.86 & 27.82 & 7.42 & 1.34 & 0.09 & 1.11 & $\mathrm{He}$ & $\mathrm{He}$ \\
\hline 54 & $37 F-1,226.30-226.35$ & 46.57 & 49.53 & 3.90 & 4.45 & 1.46 & 0.26 & 1.19 & $M$ & $\operatorname{Tr}$ \\
\hline 55 & $39 F-1,236.21-236.26$ & 77.57 & 18.83 & 3.07 & 3.11 & 1.51 & 0.30 & 1.13 & $M$ & $\operatorname{Tr}$ \\
\hline 56 & $43 F-1,255.45-255.49$ & 10.04 & 74.32 & 15.64 & 6.30 & 1.79 & 0.15 & 1.05 & $\mathrm{He}$ & $\mathrm{He}$ \\
\hline 57 & $43 F-2,256.65-256.66$ & 2.29 & 71.37 & 26.34 & 7.07 & 1.69 & 0.07 & 0.98 & $\mathrm{~F}$ & $\operatorname{Tr}$ \\
\hline 58 & $43 F-2,256.74-256.75$ & 24.27 & 65.22 & 10.51 & 5.51 & 1.85 & 0.23 & 0.98 & B & $\operatorname{Tr}$ \\
\hline 59 & $45 F-1,264.90-264.92$ & 1.81 & 70.36 & 27.83 & 7.31 & 1.53 & 0.06 & 1.10 & $\mathrm{~F}$ & $\operatorname{Tr}$ \\
\hline 60 & $45 F-1,264.95-264.97$ & 13.31 & 77.27 & 9.42 & 5.72 & 1.58 & 0.24 & 1.19 & B & $\operatorname{Tr}$ \\
\hline 61 & $47 F-1,274.60-274.65$ & 5.38 & 76.05 & 18.57 & 6.54 & 1.77 & 0.24 & 1.09 & B & $\operatorname{Tr}$ \\
\hline 62 & $49 F-2,285.27-285.31$ & 88.69 & 11.04 & 0.24 & 2.53 & 1.19 & 0.27 & 0.98 & M & $\operatorname{Tr}$ \\
\hline 63 & 51F-1, 292.85-292.89 & 0.96 & 78.42 & 20.62 & 7.03 & 1.42 & 0.12 & 1.11 & M & $\operatorname{Tr}$ \\
\hline 64 & 51F-2, 295.13-295.14 & 19.88 & 75.06 & 5.06 & 5.32 & 1.45 & 0.08 & 1.17 & $\mathrm{~T}$ & $\operatorname{Tr}$ \\
\hline
\end{tabular}


Table T3 (continued).

\begin{tabular}{|c|c|c|c|c|c|c|c|c|c|c|}
\hline $\begin{array}{c}\text { Serial } \\
\text { number }\end{array}$ & $\begin{array}{l}\text { Core, section, } \\
\text { interval }(\mathrm{m})\end{array}$ & $\begin{array}{l}\text { Sand } \\
\text { (wt\%) }\end{array}$ & $\begin{array}{c}\text { Silt } \\
\text { (wt\%) }\end{array}$ & $\begin{array}{c}\text { Clay } \\
\text { (wt\%) }\end{array}$ & $M_{\mathrm{z}}$ & $\sigma$ & SK & $K_{\mathrm{G}}$ & $\begin{array}{c}\text { Bed } \\
\text { position }\end{array}$ & $\begin{array}{l}\mathrm{Tr} / \\
\mathrm{He}\end{array}$ \\
\hline 65 & $51 \mathrm{~F}-2,295.28-295.29$ & 63.91 & 35.56 & 0.53 & 3.80 & 1.26 & 0.07 & 1.13 & M & $\mathrm{Tr}$ \\
\hline 66 & 51F-2, 295.44-295.45 & 66.97 & 31.90 & 1.13 & 3.74 & 1.22 & 0.02 & 1.24 & B & $\operatorname{Tr}$ \\
\hline 67 & $52 \mathrm{~F}-2,299.45-299.50$ & 0.23 & 74.33 & 25.44 & 7.36 & 1.30 & 0.15 & 1.14 & $\mathrm{He}$ & $\mathrm{He}$ \\
\hline 68 & $56 \mathrm{~F}-1,316.66-316.67$ & 21.66 & 71.92 & 6.42 & 5.28 & 1.50 & 0.26 & 1.16 & $\mathrm{~F}$ & $\operatorname{Tr}$ \\
\hline 69 & $56 F-1,316.78-316.79$ & 21.93 & 70.98 & 7.09 & 5.36 & 1.58 & 0.22 & 1.10 & M & $\operatorname{Tr}$ \\
\hline 70 & $56 \mathrm{~F}-1,316.89-316.90$ & 20.40 & 73.61 & 5.99 & 5.31 & 1.48 & 0.22 & 1.16 & B & $\operatorname{Tr}$ \\
\hline 71 & $60 \mathrm{~F}-1,335.49-335.50$ & 46.18 & 51.13 & 2.69 & 4.38 & 1.48 & 0.15 & 1.19 & $\mathrm{~T}$ & $\operatorname{Tr}$ \\
\hline 72 & $60 \mathrm{~F}-1,335.60-335.61$ & 52.85 & 45.62 & 1.53 & 4.16 & 1.36 & 0.09 & 1.21 & M & $\operatorname{Tr}$ \\
\hline 73 & $60 F-1,335.78-335.79$ & 43.02 & 53.10 & 3.88 & 4.55 & 1.60 & 0.18 & 1.16 & B & $\operatorname{Tr}$ \\
\hline 74 & $62 \mathrm{~F}-1,345.59-345.63$ & 4.21 & 75.13 & 20.66 & 6.94 & 1.57 & 0.09 & 1.14 & $\mathrm{He}$ & $\mathrm{He}$ \\
\hline 75 & $66 \mathrm{~F}-1,363.73-363.74$ & 0.97 & 59.35 & 39.68 & 7.78 & 1.58 & -0.00 & 1.09 & $\mathrm{~F}$ & $\operatorname{Tr}$ \\
\hline 76 & $66 \mathrm{~F}-1,363.83-363.84$ & 62.45 & 35.19 & 2.12 & 3.70 & 1.66 & 0.19 & 0.92 & $\mathrm{~T}$ & $\operatorname{Tr}$ \\
\hline 77 & $66 \mathrm{~F}-1,364.00-364.01$ & 39.01 & 54.35 & 6.61 & 4.84 & 1.86 & 0.19 & 0.96 & M & $\operatorname{Tr}$ \\
\hline 78 & $66 \mathrm{~F}-1,364.16-364.17$ & 38.69 & 52.82 & 8.48 & 4.98 & 1.98 & 0.23 & 0.95 & B & $\operatorname{Tr}$ \\
\hline 79 & 70F-3, 386.01-386.03 & 0.61 & 73.00 & 26.39 & 7.25 & 1.49 & 0.09 & 1.06 & $\mathrm{~F}$ & $\operatorname{Tr}$ \\
\hline 80 & 70F-3, 386.04-386.06 & 22.58 & 67.05 & 10.37 & 5.60 & 1.86 & 0.13 & 1.01 & $\mathrm{~T}$ & $\operatorname{Tr}$ \\
\hline 81 & $74 \mathrm{~F}-2,403.56-403.61$ & 13.81 & 78.67 & 7.52 & 5.61 & 1.48 & 0.18 & 1.25 & M & $\operatorname{Tr}$ \\
\hline 82 & $76 \mathrm{~F}-1,411.82-411.83$ & 11.80 & 73.12 & 15.08 & 6.06 & 1.86 & 0.25 & 0.97 & $\mathrm{~F}$ & $\operatorname{Tr}$ \\
\hline 83 & $76 F-1,411.90-411.91$ & 16.40 & 82.01 & 1.59 & 5.05 & 0.98 & 0.12 & 1.32 & $\mathrm{~T}$ & $\operatorname{Tr}$ \\
\hline 84 & $76 \mathrm{~F}-1,412.04-412.05$ & 29.35 & 66.51 & 4.14 & 4.89 & 1.44 & 0.16 & 1.28 & B & $\operatorname{Tr}$ \\
\hline 85 & $82 F-1,439.78-439.82$ & 1.25 & 68.31 & 30.44 & 7.45 & 1.50 & 0.04 & 1.13 & $\mathrm{He}$ & $\mathrm{He}$ \\
\hline 86 & $84 \mathrm{~F}-1,449.39-449.40$ & 8.85 & 82.75 & 8.40 & 5.90 & 1.43 & 0.15 & 1.14 & $\mathrm{~F}$ & $\operatorname{Tr}$ \\
\hline 87 & $84 \mathrm{~F}-1,449.49-449.50$ & 39.52 & 59.26 & 1.22 & 4.49 & 1.15 & 0.15 & 1.19 & M & $\operatorname{Tr}$ \\
\hline 88 & $84 \mathrm{~F}-1,449.58-449.59$ & 29.47 & 68.45 & 2.09 & 4.77 & 1.19 & 0.13 & 1.20 & B & $\operatorname{Tr}$ \\
\hline 89 & $86 \mathrm{~F}-1,459.35-459.37$ & 1.19 & 75.30 & 23.51 & 7.11 & 1.51 & 0.08 & 1.10 & $\mathrm{~F}$ & $\operatorname{Tr}$ \\
\hline 90 & $86 F-1,459.37-459.39$ & 4.17 & 83.83 & 11.99 & 6.40 & 1.46 & 0.20 & 1.37 & T & $\operatorname{Tr}$ \\
\hline 91 & $94 \mathrm{~F}-1,497.46-497.51$ & 52.68 & 40.01 & 7.28 & 4.43 & 2.00 & 0.36 & 1.03 & M & $\mathrm{Tr}$ \\
\hline 92 & $100 \mathrm{~F}-1,525.43-525.44$ & 1.33 & 66.74 & 31.93 & 7.47 & 1.50 & 0.00 & 1.10 & $\mathrm{~F}$ & $\operatorname{Tr}$ \\
\hline 93 & $100 \mathrm{~F}-1,525.67-525.68$ & 26.33 & 66.80 & 6.87 & 5.17 & 1.67 & 0.18 & 1.19 & $\mathrm{~T}$ & $\operatorname{Tr}$ \\
\hline 94 & $100 \mathrm{~F}-1,525.86-525.87$ & 28.14 & 69.20 & 2.65 & 4.66 & 1.11 & 0.08 & 1.40 & B & $\mathrm{Tr}$ \\
\hline 95 & 100F-1, 526.10-526.15 & 1.07 & 77.18 & 21.75 & 7.04 & 1.47 & 0.11 & 1.08 & $\mathrm{~F}$ & $\operatorname{Tr}$ \\
\hline 96 & $106 \mathrm{~F}-1,554.55-554.60$ & 57.19 & 39.27 & 3.54 & 3.95 & 1.68 & 0.14 & 1.10 & M & $\operatorname{Tr}$ \\
\hline 97 & $111 \mathrm{X}-1,574.00-574.01$ & 1.08 & 65.77 & 33.15 & 7.37 & 1.75 & 0.04 & 0.97 & $\mathrm{~F}$ & $\operatorname{Tr}$ \\
\hline 98 & $111 \mathrm{X}-1,574.26-574.27$ & 13.47 & 75.46 & 11.08 & 5.92 & 1.67 & 0.13 & 1.08 & M & $\operatorname{Tr}$ \\
\hline 99 & $111 X-1,574.42-574.43$ & 78.76 & 19.30 & 0.99 & 3.03 & 1.44 & 0.20 & 1.09 & B & $\operatorname{Tr}$ \\
\hline 100 & $111 X-3,577.39-577.44$ & 32.35 & 60.80 & 6.85 & 4.93 & 1.74 & 0.18 & 1.16 & $\mathrm{~T}$ & $\operatorname{Tr}$ \\
\hline
\end{tabular}

Table T4. Grain size analysis data, Hole U1451B. $M_{z}=$ mean, $\sigma=$ sorting (standard deviation), $S K=$ skewness, $K_{\mathrm{G}}=$ kurtosis. Bed position: $\mathrm{F}=$ fine (upper muddy part of turbidite), $\mathrm{T}=$ top, $\mathrm{M}=$ middle, $\mathrm{B}=$ base, respectively (lower sandy part of turbidite). $\mathrm{Tr}=$ turbidite, $\mathrm{He}=$ hemipelagic mud. Color blocks $=$ individual turbidite sequences and the differing horizons within them. An expanded version of this table is available in GRAINSIZE in Supplementary material. (Continued on next page.) Download table in CSV format.

\begin{tabular}{|c|c|c|c|c|c|c|c|c|c|c|}
\hline $\begin{array}{c}\text { Serial } \\
\text { number }\end{array}$ & $\begin{array}{l}\text { Core, section, } \\
\text { interval }(\mathrm{m})\end{array}$ & $\begin{array}{l}\text { Sand } \\
\text { (wt\%) }\end{array}$ & $\begin{array}{c}\text { Silt } \\
\text { (wt\%) }\end{array}$ & $\begin{array}{l}\text { Clay } \\
\text { (wt \%) }\end{array}$ & $M_{z}$ & $\sigma$ & SK & $K_{\mathrm{G}}$ & $\begin{array}{c}\text { Bed } \\
\text { position }\end{array}$ & $\begin{array}{l}\mathrm{Tr} / \\
\mathrm{He}\end{array}$ \\
\hline \multicolumn{11}{|c|}{ 354-U1451B- } \\
\hline 1 & $3 X-1,551.97-552.00$ & 45.57 & 46.78 & 7.64 & 4.66 & 2.02 & 0.26 & 1.05 & B & $\operatorname{Tr}$ \\
\hline 2 & $3 X-1,552.00-552.03$ & 0.27 & 66.89 & 32.84 & 7.42 & 1.59 & 0.01 & 1.00 & $\mathrm{~T}$ & $\operatorname{Tr}$ \\
\hline 3 & $3 X-1,552.01-552.02$ & 0.61 & 66.95 & 32.44 & 7.45 & 1.59 & 0.05 & 1.01 & $\mathrm{~T}$ & $\operatorname{Tr}$ \\
\hline 4 & $3 X-1,552.07-552.08$ & 19.70 & 68.27 & 12.03 & 5.72 & 1.82 & 0.27 & 0.96 & M & $\operatorname{Tr}$ \\
\hline 5 & $3 X-1,552.13-552.15$ & 16.22 & 70.92 & 12.86 & 5.86 & 1.80 & 0.25 & 0.96 & $\mathrm{~T}$ & $\operatorname{Tr}$ \\
\hline 6 & $6 X-1,581.87-581.92$ & 0.01 & 73.98 & 26.01 & 7.39 & 1.28 & 0.16 & 1.14 & M & $\operatorname{Tr}$ \\
\hline 7 & 7X-CC, 591.21-591.27 & 21.48 & 67.04 & 11.48 & 5.64 & 1.79 & 0.33 & 0.98 & M & $\operatorname{Tr}$ \\
\hline 8 & $11 X-1,619.90-619.95$ & 0.10 & 67.29 & 32.62 & 7.48 & 1.61 & 0.12 & 0.97 & $\mathrm{~T}$ & $\operatorname{Tr}$ \\
\hline 9 & $12 X-1,629.30-629.35$ & 0.07 & 64.20 & 35.73 & 7.67 & 1.45 & 0.08 & 1.03 & $\mathrm{~F}$ & $\operatorname{Tr}$ \\
\hline 10 & $16 R-1,655.66-655.67$ & 0.26 & 69.96 & 29.79 & 7.36 & 1.53 & 0.13 & 0.97 & $\mathrm{~F}$ & $\operatorname{Tr}$ \\
\hline 11 & 16R-1, 655.78-655.79 & 31.13 & 57.86 & 11.01 & 5.37 & 1.96 & 0.31 & 0.98 & $\mathrm{~T}$ & $\operatorname{Tr}$ \\
\hline 12 & $16 R-2,657.48-657.53$ & 31.08 & 57.95 & 10.98 & 5.35 & 1.97 & 0.31 & 1.02 & M & $\operatorname{Tr}$ \\
\hline 13 & $19 R-1,685.66-685.70$ & 31.23 & 56.49 & 12.28 & 5.42 & 2.04 & 0.32 & 0.96 & M & $\operatorname{Tr}$ \\
\hline 14 & 21R-1, 704.73-704.74 & 0.71 & 67.22 & 32.06 & 7.49 & 1.50 & 0.05 & 1.07 & $\mathrm{~F}$ & $\operatorname{Tr}$ \\
\hline 15 & 21R-1, 704.85-704.86 & 7.51 & 67.99 & 24.50 & 6.77 & 1.91 & 0.02 & 0.92 & M & $\operatorname{Tr}$ \\
\hline 16 & 21R-1, 705.08-705.09 & 41.77 & 52.69 & 5.54 & 4.71 & 1.69 & 0.25 & 1.07 & B & $\operatorname{Tr}$ \\
\hline 17 & $21 \mathrm{R}-2,707.20-707.30$ & 1.26 & 63.21 & 35.54 & 7.57 & 1.60 & 0.00 & 1.04 & $\mathrm{~F}$ & $\operatorname{Tr}$ \\
\hline 18 & $23 R-1,725.10-725.15$ & 44.23 & 49.86 & 5.91 & 4.64 & 1.75 & 0.27 & 1.11 & B & $\operatorname{Tr}$ \\
\hline 19 & $23 \mathrm{R}-1,725.17-725.22$ & 4.02 & 65.36 & 30.62 & 7.20 & 1.83 & 0.03 & 0.97 & $\mathrm{~F}$ & $\operatorname{Tr}$ \\
\hline 20 & 27R-1, 763.70-763.71 & 27.46 & 59.07 & 13.47 & 5.63 & 2.10 & 0.14 & 0.88 & $\mathrm{~T}$ & $\operatorname{Tr}$ \\
\hline 21 & 27R-1, 763.90-763.91 & 53.21 & 39.72 & 7.06 & 4.29 & 2.15 & 0.29 & 0.96 & M & $\operatorname{Tr}$ \\
\hline 22 & 27R-1, 764.10-764.11 & 37.72 & 52.22 & 10.06 & 5.10 & 2.02 & 0.28 & 0.95 & B & $\mathrm{Tr}$ \\
\hline
\end{tabular}


Table T4 (continued).

\begin{tabular}{|c|c|c|c|c|c|c|c|c|c|c|}
\hline $\begin{array}{c}\text { Serial } \\
\text { number }\end{array}$ & $\begin{array}{l}\text { Core, section, } \\
\text { interval }(\mathrm{m})\end{array}$ & $\begin{array}{l}\text { Sand } \\
\text { (wt\%) }\end{array}$ & $\begin{array}{c}\text { Silt } \\
\text { (wt\%) }\end{array}$ & $\begin{array}{l}\text { Clay } \\
\text { (wt\%) }\end{array}$ & $M_{z}$ & $\sigma$ & SK & $K_{\mathrm{G}}$ & $\begin{array}{c}\text { Bed } \\
\text { position }\end{array}$ & $\begin{array}{l}\mathrm{Tr} / \\
\mathrm{He}\end{array}$ \\
\hline 23 & 27R-2, 764.91-764.95 & 12.95 & 60.56 & 26.49 & 6.60 & 2.15 & 0.10 & 0.78 & $\mathrm{~F}$ & $\operatorname{Tr}$ \\
\hline 24 & $29 \mathrm{R}-1,782.99-783.00$ & 0.30 & 67.85 & 31.85 & 7.60 & 1.28 & 0.09 & 1.11 & $\mathrm{~F}$ & $\operatorname{Tr}$ \\
\hline 25 & 29R-1, 783.08-783.09 & 64.86 & 31.47 & 3.67 & 3.65 & 1.88 & 0.24 & 1.07 & T & $\operatorname{Tr}$ \\
\hline 26 & $29 R-1,783.30-783.31$ & 56.02 & 38.83 & 5.15 & 4.14 & 1.83 & 0.27 & 1.11 & M & $\operatorname{Tr}$ \\
\hline 27 & $29 \mathrm{R}-1,783.49-783.50$ & 61.25 & 34.66 & 4.09 & 3.87 & 1.84 & 0.26 & 1.08 & B & $\operatorname{Tr}$ \\
\hline 28 & $31 \mathrm{R}-1,802.67-802.69$ & 0.23 & 68.24 & 31.53 & 7.43 & 1.56 & 0.11 & 0.99 & $\mathrm{~F}$ & $\operatorname{Tr}$ \\
\hline 29 & $31 \mathrm{R}-2,803.32-803.34$ & 47.26 & 48.40 & 4.34 & 4.47 & 1.57 & 0.27 & 1.08 & M & $\operatorname{Tr}$ \\
\hline 30 & $33 \mathrm{R}-1,821.43-821.48$ & 0.85 & 71.43 & 27.72 & 7.19 & 1.64 & 0.13 & 0.97 & $\mathrm{~F}$ & $\operatorname{Tr}$ \\
\hline 31 & $33 R-2,822.66-822.71$ & 22.02 & 64.16 & 13.83 & 5.71 & 1.93 & 0.33 & 0.92 & M & $\operatorname{Tr}$ \\
\hline 32 & 34R-1, 831.17-831.18 & 1.75 & 76.95 & 21.30 & 6.77 & 1.64 & 0.14 & 0.91 & $\mathrm{~F}$ & $\operatorname{Tr}$ \\
\hline 33 & $34 \mathrm{R}-1,831.23-831.24$ & 16.42 & 67.86 & 15.73 & 6.08 & 1.93 & 0.14 & 0.92 & T & $\operatorname{Tr}$ \\
\hline 34 & $34 \mathrm{R}-1,831.30-831.31$ & 21.13 & 65.95 & 12.92 & 5.79 & 1.89 & 0.19 & 0.90 & M & $\operatorname{Tr}$ \\
\hline 35 & $34 \mathrm{R}-1,831.38-831.39$ & 24.84 & 62.62 & 12.55 & 5.64 & 1.98 & 0.19 & 0.94 & B & $\operatorname{Tr}$ \\
\hline 36 & 36R-CC, 851.88-851.91 & 0.64 & 65.03 & 34.34 & 7.55 & 1.49 & 0.01 & 1.05 & $\mathrm{~F}$ & $\operatorname{Tr}$ \\
\hline 37 & 37R-3, 863.02-863.03 & 0.19 & 72.62 & 27.19 & 7.44 & 1.24 & 0.10 & 1.09 & $\mathrm{~F}$ & $\operatorname{Tr}$ \\
\hline 38 & 37R-3, 863.15-863.17 & 44.70 & 49.14 & 6.17 & 4.72 & 1.87 & 0.27 & 0.91 & $\mathrm{~T}$ & $\operatorname{Tr}$ \\
\hline 39 & $37 \mathrm{R}-3,863.26-863.27$ & 56.22 & 36.19 & 7.59 & 4.27 & 2.29 & 0.34 & 0.92 & B & $\operatorname{Tr}$ \\
\hline 40 & 42R-1, 908.96-908.97 & 0.62 & 65.56 & 33.82 & 7.64 & 1.34 & -0.00 & 1.18 & $\mathrm{~F}$ & $\operatorname{Tr}$ \\
\hline 41 & $42 R-1,909.04-909.05$ & 10.98 & 79.24 & 9.78 & 5.87 & 1.54 & 0.20 & 1.13 & $\mathrm{~T}$ & $\operatorname{Tr}$ \\
\hline 42 & $42 R-1,909.09-909.10$ & 9.13 & 85.02 & 5.85 & 5.68 & 1.27 & 0.16 & 1.26 & M & $\operatorname{Tr}$ \\
\hline 43 & 42R-1, 909.18-909.19 & 42.43 & 51.74 & 5.83 & 4.74 & 1.86 & 0.20 & 0.89 & B & $\operatorname{Tr}$ \\
\hline 44 & $45 \mathrm{R}-2,938.90-938.91$ & 0.14 & 55.72 & 44.14 & 8.01 & 1.24 & 0.06 & 1.07 & $\mathrm{~F}$ & $\operatorname{Tr}$ \\
\hline 45 & $45 \mathrm{R}-2,938.98-938.99$ & 21.60 & 67.45 & 10.95 & 5.65 & 1.82 & 0.20 & 0.97 & T & $\operatorname{Tr}$ \\
\hline 46 & 45R-2, 939.17-939.18 & 24.15 & 69.95 & 5.90 & 5.21 & 1.49 & 0.23 & 1.13 & M & $\operatorname{Tr}$ \\
\hline 47 & $45 R-2,939.27-939.28$ & 31.24 & 64.15 & 4.60 & 4.93 & 1.48 & 0.21 & 1.14 & B & $\operatorname{Tr}$ \\
\hline 48 & $45 R-2,939.30-939.31$ & 0.26 & 61.84 & 37.89 & 7.75 & 1.41 & 0.05 & 1.05 & $\mathrm{~F}$ & $\operatorname{Tr}$ \\
\hline 49 & $45 R-2,939.41-939.42$ & 8.71 & 73.43 & 17.85 & 6.42 & 1.81 & 0.11 & 0.94 & M & $\operatorname{Tr}$ \\
\hline 50 & $45 R-2,939.61-939.62$ & 14.50 & 70.14 & 15.36 & 6.07 & 1.86 & 0.20 & 0.91 & B & $\operatorname{Tr}$ \\
\hline 51 & 50R-1, 977.94-977.95 & 0.78 & 71.94 & 27.28 & 7.26 & 1.51 & 0.07 & 1.03 & $\mathrm{~F}$ & $\operatorname{Tr}$ \\
\hline 52 & 50R-1, 977.99-978.00 & 3.62 & 87.08 & 9.30 & 6.23 & 1.30 & 0.17 & 1.14 & T & $\operatorname{Tr}$ \\
\hline 53 & 50R-1, 978.09-978.10 & 4.38 & 86.32 & 9.30 & 6.18 & 1.33 & 0.16 & 1.15 & M & $\operatorname{Tr}$ \\
\hline 54 & 50R-1, 978.26-978.27 & 10.14 & 81.98 & 7.88 & 5.83 & 1.43 & 0.14 & 1.18 & B & $\operatorname{Tr}$ \\
\hline 55 & 51R-1, 987.65-987.66 & 0.86 & 71.67 & 27.47 & 7.23 & 1.57 & 0.09 & 1.02 & $\mathrm{~F}$ & $\operatorname{Tr}$ \\
\hline 56 & 51R-1, 987.68-987.69 & 19.27 & 76.49 & 4.23 & 5.19 & 1.27 & 0.24 & 1.17 & T & $\operatorname{Tr}$ \\
\hline 57 & 51R-1, 987.81-987.82 & 29.02 & 66.06 & 4.92 & 5.01 & 1.46 & 0.24 & 1.12 & B & $\operatorname{Tr}$ \\
\hline 58 & $51 R-2,988.57-988.58$ & 0.41 & 66.40 & 33.19 & 7.51 & 1.50 & 0.04 & 1.03 & $\mathrm{~F}$ & $\operatorname{Tr}$ \\
\hline 59 & 51R-2, 988.65-988.66 & 17.94 & 73.82 & 8.24 & 5.52 & 1.56 & 0.27 & 1.10 & T & $\operatorname{Tr}$ \\
\hline 60 & 51R-2, 988.78-988.79 & 35.68 & 57.22 & 7.10 & 5.00 & 1.74 & 0.28 & 1.07 & M & $\operatorname{Tr}$ \\
\hline 61 & 51R-2, 989.10-989.11 & 60.15 & 35.98 & 3.87 & 3.97 & 1.78 & 0.27 & 1.09 & B & $\operatorname{Tr}$ \\
\hline 62 & 54R-1, 1016.40-1016.41 & 20.84 & 72.67 & 6.49 & 5.31 & 1.48 & 0.26 & 1.14 & T & $\operatorname{Tr}$ \\
\hline 63 & 54R-1, 1016.69-1016.70 & 18.99 & 70.62 & 10.39 & 5.64 & 1.71 & 0.27 & 0.99 & M & $\operatorname{Tr}$ \\
\hline 64 & 54R-1, 1016.96-1016.97 & 19.50 & 71.24 & 9.25 & 5.61 & 1.67 & 0.20 & 1.01 & B & $\operatorname{Tr}$ \\
\hline 65 & 54R-2, 1017.01-1017.03 & 0.05 & 61.79 & 38.16 & 7.70 & 1.46 & 0.01 & 1.02 & $\mathrm{~F}$ & $\operatorname{Tr}$ \\
\hline 66 & 54R-2, 1017.17-1017.18 & 14.31 & 77.39 & 8.30 & 5.66 & 1.53 & 0.23 & 1.08 & T & $\operatorname{Tr}$ \\
\hline 67 & 54R-2, 1017.28-1017.29 & 17.30 & 75.90 & 6.81 & 5.47 & 1.48 & 0.22 & 1.10 & M & $\operatorname{Tr}$ \\
\hline 68 & 54R-2, 1017.42-1017.43 & 16.50 & 77.69 & 5.81 & 5.43 & 1.40 & 0.23 & 1.10 & B & $\operatorname{Tr}$ \\
\hline 69 & 55R-1, 1025.64-1025.65 & 4.65 & 59.48 & 35.87 & 7.54 & 1.66 & -0.12 & 1.22 & $\mathrm{~F}$ & $\operatorname{Tr}$ \\
\hline 70 & $55 \mathrm{R}-1,1025.71-1025.72$ & 5.42 & 81.98 & 12.60 & 6.33 & 1.50 & 0.14 & 1.10 & $\mathrm{~T}$ & $\operatorname{Tr}$ \\
\hline 71 & 55R-1, 1025.87-1025.88 & 49.88 & 43.88 & 6.24 & 4.52 & 1.83 & 0.35 & 1.00 & M & $\operatorname{Tr}$ \\
\hline 72 & $55 \mathrm{R}-1,1026.05-1026.06$ & 44.17 & 49.67 & 6.16 & 4.72 & 1.79 & 0.29 & 1.00 & B & $\operatorname{Tr}$ \\
\hline 73 & 57R-2, 1040.97-1040.99 & 0.17 & 54.32 & 45.51 & 8.02 & 1.35 & -0.02 & 1.17 & $\mathrm{~F}$ & $\operatorname{Tr}$ \\
\hline 74 & 57R-2, 1041.12-1041.13 & 25.10 & 66.40 & 8.51 & 5.34 & 1.67 & 0.31 & 1.08 & M & $\operatorname{Tr}$ \\
\hline 75 & 57R-2, 1041.29-1041.30 & 25.77 & 65.56 & 8.66 & 5.33 & 1.70 & 0.31 & 1.07 & M & $\operatorname{Tr}$ \\
\hline 76 & 57R-2, 1041.53-1041.54 & 32.19 & 60.43 & 7.38 & 5.09 & 1.70 & 0.29 & 1.08 & B & $\operatorname{Tr}$ \\
\hline 77 & $58 \mathrm{R}-1,1045.28-1045.29$ & 1.92 & 76.37 & 21.71 & 7.08 & 1.42 & 0.02 & 1.10 & $\mathrm{~F}$ & $\operatorname{Tr}$ \\
\hline 78 & 58R-1, 1045.31-1045.32 & 17.27 & 73.39 & 9.33 & 5.72 & 1.68 & 0.12 & 1.06 & T & $\operatorname{Tr}$ \\
\hline 79 & $58 \mathrm{R}-1,1045.45-1045.46$ & 46.23 & 49.46 & 4.31 & 4.39 & 1.61 & 0.17 & 1.22 & M & $\operatorname{Tr}$ \\
\hline 80 & 58R-1, 1045.46-1045.47 & 39.38 & 55.38 & 5.24 & 4.66 & 1.55 & 0.26 & 1.30 & M & $\operatorname{Tr}$ \\
\hline 81 & 59R-2, 1056.52-1056.53 & 0.46 & 63.95 & 35.59 & 7.58 & 1.52 & -0.01 & 1.04 & $\mathrm{~F}$ & $\operatorname{Tr}$ \\
\hline 82 & 59R-2, 1056.55-1056.56 & 42.56 & 51.26 & 6.18 & 4.69 & 1.76 & 0.24 & 1.08 & $\mathrm{~T}$ & $\operatorname{Tr}$ \\
\hline 83 & $59 R-2,1056.80-1056.81$ & 56.44 & 39.37 & 4.19 & 4.08 & 1.75 & 0.26 & 1.02 & M & $\operatorname{Tr}$ \\
\hline 84 & $59 R-2,1057.10-1057.11$ & 42.52 & 50.80 & 6.68 & 4.73 & 1.75 & 0.31 & 1.19 & B & $\operatorname{Tr}$ \\
\hline 85 & 60R-2, 1066.31-1066.32 & 1.85 & 76.67 & 21.48 & 6.79 & 1.66 & 0.18 & 0.95 & $\mathrm{~F}$ & $\operatorname{Tr}$ \\
\hline 86 & $60 \mathrm{R}-3,1067.49-1067.50$ & 0.56 & 68.01 & 31.43 & 7.46 & 1.47 & 0.04 & 1.05 & $\mathrm{~F}$ & $\operatorname{Tr}$ \\
\hline 87 & $60 R-3,1067.62-1067.63$ & 15.57 & 75.50 & 8.93 & 5.58 & 1.56 & 0.30 & 1.13 & $\mathrm{~T}$ & $\operatorname{Tr}$ \\
\hline 88 & 60R-3, 1067.77-1067.78 & 26.59 & 66.02 & 7.38 & 5.23 & 1.61 & 0.28 & 1.09 & M & $\operatorname{Tr}$ \\
\hline 89 & 60R-3, 1067.93-1067.94 & 25.82 & 66.69 & 7.49 & 5.25 & 1.60 & 0.29 & 1.11 & B & $\operatorname{Tr}$ \\
\hline 90 & $62 \mathrm{R}-1,1084.71-1084.72$ & 29.90 & 60.68 & 9.42 & 5.28 & 1.81 & 0.30 & 1.03 & $\mathrm{~T}$ & $\operatorname{Tr}$ \\
\hline 91 & 62R-1, 1085.10-1085.11 & 23.75 & 67.39 & 8.87 & 5.40 & 1.68 & 0.30 & 1.07 & M & $\operatorname{Tr}$ \\
\hline
\end{tabular}


(massive) beds (Figure F4). The frequency curves show that inversely graded beds are dominant at Site U1450, whereas thicker massive beds dominate at Site U1451 among the analyzed beds. Most of the inversely graded beds show a coarsening-upward trend from their base to their middle horizons and then show normal grading toward the top horizons in the sandy parts. Overall from the analyzed turbidite beds, $38 \%$ are normally graded (examples in Figure F4A), 29\% are inversely graded (Figure F4B), and 33\% are massive beds (Figure F4C).

Figure F3. Ternary sand-silt-clay plots (after Shepard, 1954). A. Site U1450. B. Site U1451.

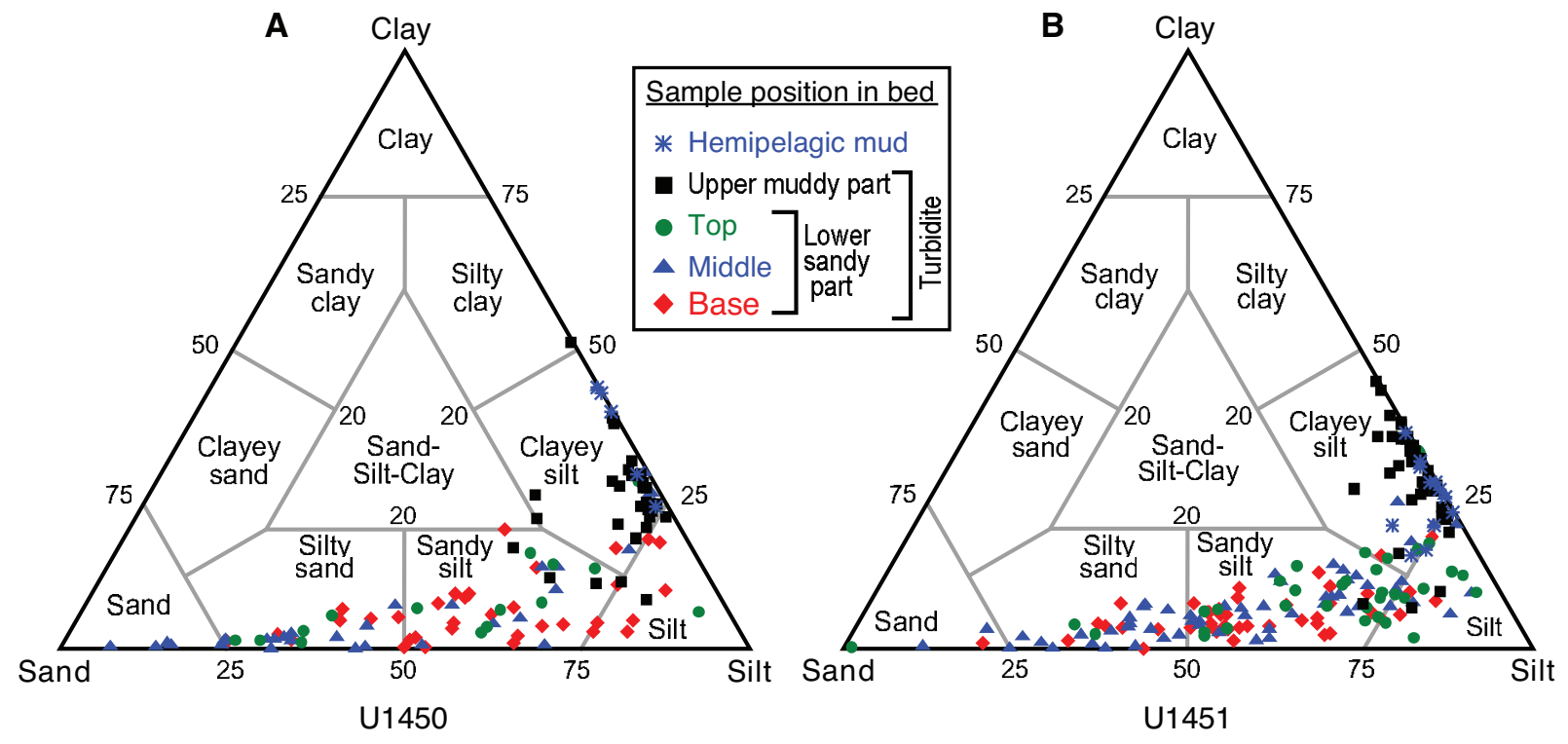

Figure F4. Frequency curve plots against grain size $(\phi)$ showing examples of differing bed types. A. Normally graded beds. B. Inversely graded beds. C. Massive (homogeneous) beds. (Continued on next page.)

A

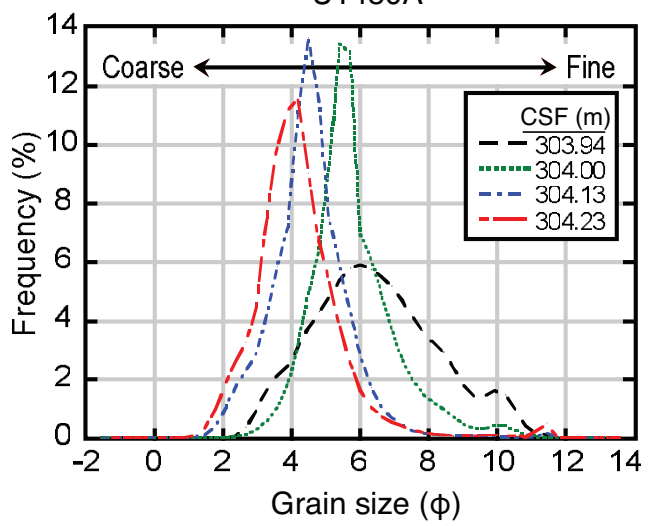

B

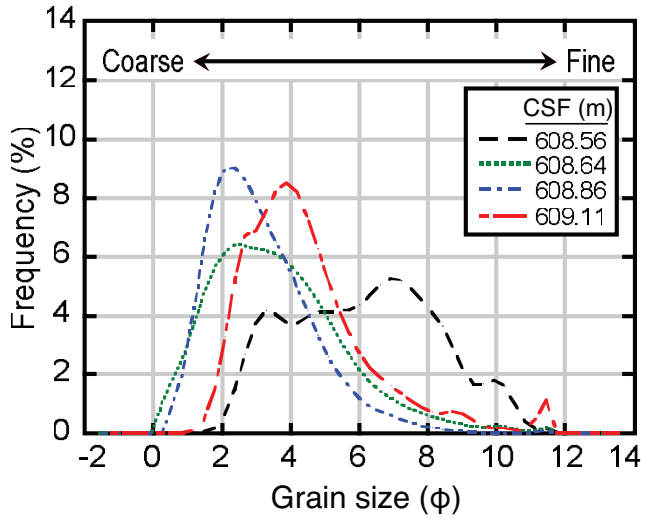

U1450B

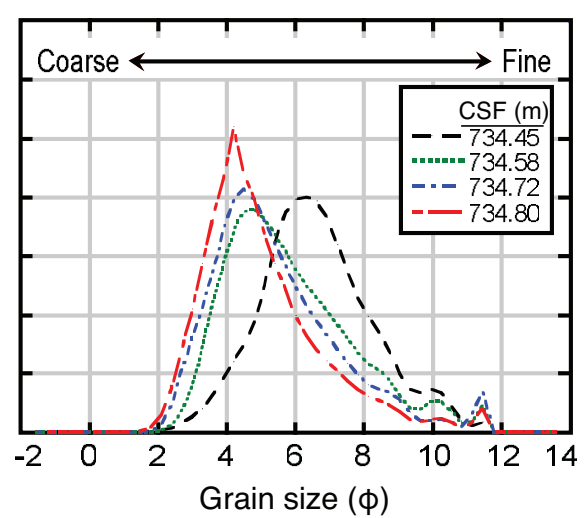

U1450B

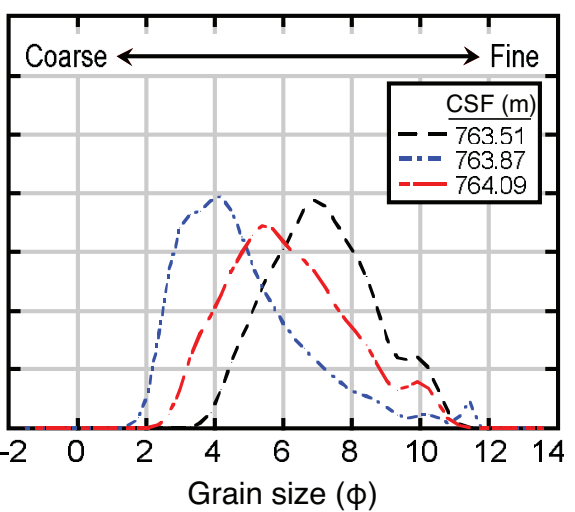

U1451B

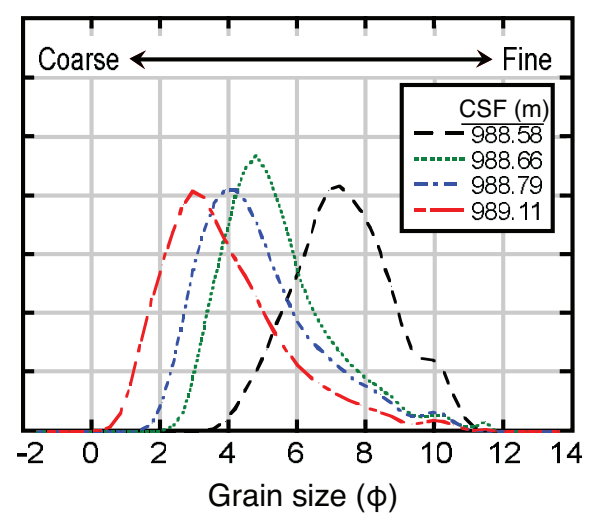

U1451A

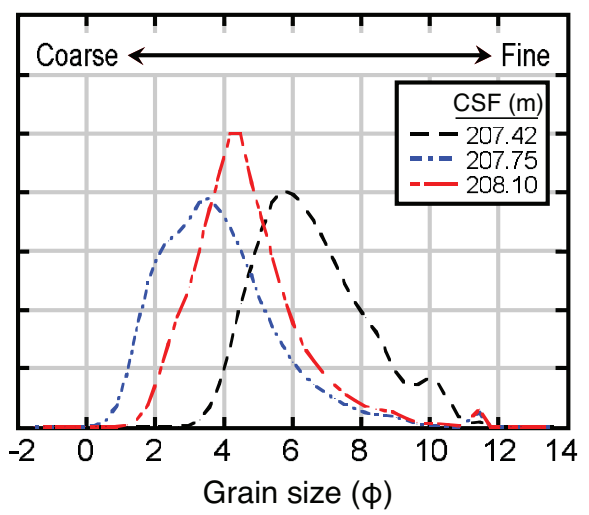


Figure F4 (continued).
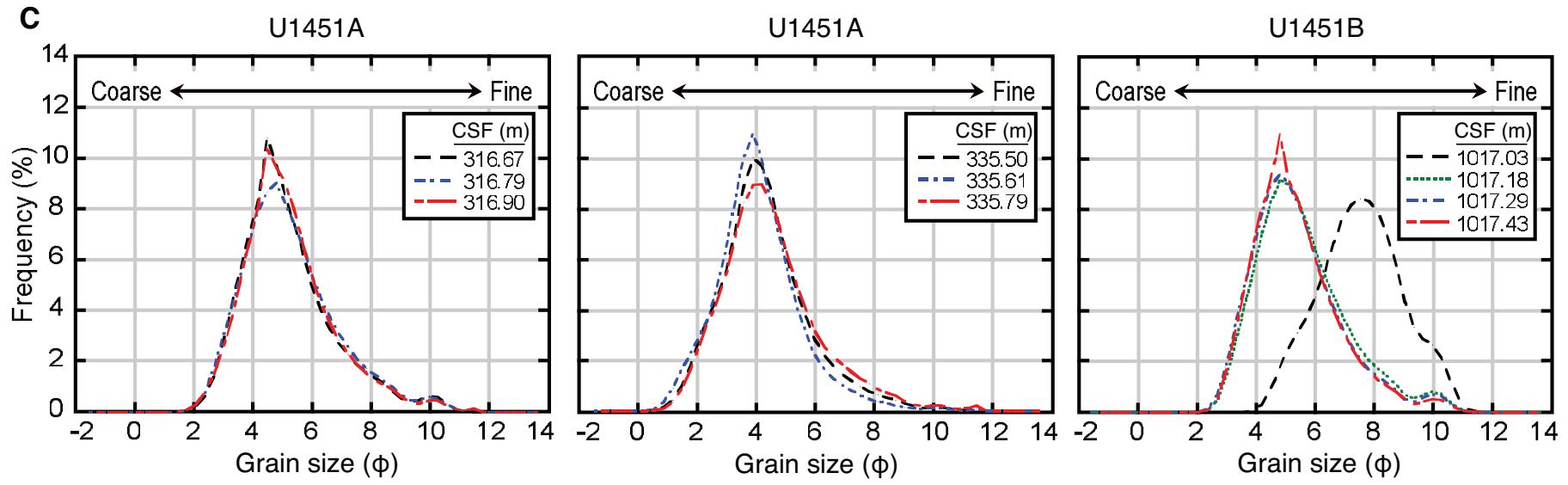

\begin{tabular}{|l|l|}
\hline Sample position in bed & ---- Mud \\
Upper muddy part & Lower sandy part \\
\hline
\end{tabular}

\section{Acknowledgments}

We thank the International Ocean Discovery Program (IODP) and Kochi Core Center (KCC) staff for their help and support during our sediment sampling. We thank Dr. M. Lupker for constructive review of the manuscript, Dr. B.P. Roser for critical reading and helpful comments on an early version of the manuscript, Dr. P. Huyghe for providing additional samples as well as her fruitful suggestions on research collaboration, and Drs. H. Lantzsch and M.E. Weber for their valuable suggestions during sample collection. Special thanks are due to Drs. C. France-Lanord, V. Spiess, A. Klaus, T. Schwenk, and the scientists of Expedition 354 for providing the opportunity to contribute this paper to the IODP publication and for collaboration during onshore and postcruise meetings. This research has been financially supported in part by the Japan Drilling Earth Science Consortium (J-DESC) and the Japan Agency for Marine-Earth Science and Technology (JAMSTEC).

\section{References}

Bouma, A.H., 2000. Fine-grained, mud-rich turbidite systems: model and comparison with coarse-grained, sand-rich systems. In Bouma, A.H., and Stone, C.G. (Eds.), Fine-Grained Turbidite Systems. AAPG Memoir, 72:920. https://pubs.geoscienceworld.org/books/chapterpdf/3841040/9781629812625_ch02.pdf

Bouma, A.H., 2004. Key controls on the characteristics of turbidite systems. In Lomas, S.A., and Joseph, P. (Eds.), Confined Turbidite Systems. Geological Society Special Publication, 222:9-22. https://doi.org/10.1144/GSL.SP.2004.222.01.02

Bouma, A.H., Coleman, J.M., Stelting, C.E., and Kohl, B., 1989. Influence of relative sea level changes on the construction of the Mississippi Fan. GeoMarine Letters, 9(3):161-170. https://doi.org/10.1007/BF02431043

Dennielou, B., Huchon, A., Beaudouin, C., and Berné, S., 2006. Vertical grainsize variability within a turbidite levee: autocyclicity or allocyclicity? A case study from the Rhône neofan, Gulf of Lions, Western Mediterranean. Marine Geology, 234(1-4):191-213. https://doi.org/10.1016/j.margeo.2006.09.019

Folk, R.L., 1974. Petrology of Sedimentary Rocks: Austin, TX (University of Texas).

Folk, R.L., and Ward, W.C., 1957. Brazos River bar [Texas]: a study in the significance of grain size parameters. Journal of Sedimentary Research, 27(1):3-26. https://doi.org/10.1306/74D70646-2B21-11D78648000102C1865D
France-Lanord, C., Spiess, V., Klaus, A., Schwenk, T., Adhikari, R.R., Adhikari, S.K., Bahk, J.-J., Baxter, A.T., Cruz, J.W., Das, S.K., Dekens, P., Duleba, W., Fox, L.R., Galy, A., Galy, V., Ge, J., Gleason, J.D., Gyawali, B.R., Huyghe, P., Jia, G., Lantzsch, H., Manoj, M.C., Martos Martin, Y., Meynadier, L., Najman, Y.M.R., Nakajima, A., Ponton, C., Reilly, B.T., Rogers, K.G., Savian, J.F., Selkin, P.A., Weber, M.E., Williams, T., and Yoshida, K., 2016a. Expedition 354 summary. In France-Lanord, C., Spiess, V., Klaus, A., Schwenk, T., and the Expedition 354 Scientists, Bengal Fan. Proceedings of the International Ocean Discovery Program, 354: College Station, TX (International Ocean Discovery Program).

https://doi.org/10.14379/iodp.proc.354.101.2016

France-Lanord, C., Spiess, V., Klaus, A., Adhikari, R.R., Adhikari, S.K., Bahk, J.-J., Baxter, A.T., Cruz, J.W., Das, S.K., Dekens, P., Duleba, W., Fox, L.R., Galy, A., Galy, V., Ge, J., Gleason, J.D., Gyawali, B.R., Huyghe, P., Jia, G., Lantzsch, H., Manoj, M.C., Martos Martin, Y., Meynadier, L., Najman, Y.M.R., Nakajima, A., Ponton, C., Reilly, B.T., Rogers, K.G., Savian, J.F., Schwenk, T., Selkin, P.A., Weber, M.E., Williams, T., and Yoshida, K., 2016b. Site U1450. In France-Lanord, C., Spiess, V., Klaus, A., Schwenk, T., and the Expedition 354 Scientists, Bengal Fan. Proceedings of the International Ocean Discovery Program, 354: College Station, TX (International Ocean Discovery Program). https://doi.org/10.14379/iodp.proc.354.104.2016

France-Lanord, C., Spiess, V., Klaus, A., Adhikari, R.R., Adhikari, S.K., Bahk, J.-J., Baxter, A.T., Cruz, J.W., Das, S.K., Dekens, P., Duleba, W., Fox, L.R., Galy, A., Galy, V., Ge, J., Gleason, J.D., Gyawali, B.R., Huyghe, P., Jia, G., Lantzsch, H., Manoj, M.C., Martos Martin, Y., Meynadier, L., Najman, Y.M.R., Nakajima, A., Ponton, C., Reilly, B.T., Rogers, K.G., Savian, J.F., Schwenk, T., Selkin, P.A., Weber, M.E., Williams, T., and Yoshida, K., 2016c. Site U1451. In France-Lanord, C., Spiess, V., Klaus, A., Schwenk, T., and the Expedition 354 Scientists, Bengal Fan. Proceedings of the International Ocean Discovery Program, 354: College Station, TX (International Ocean Discovery Program).

https://doi.org/10.14379/iodp.proc.354.105.2016

Gorsline, D.S., 1984. A review of fine-grained sediment origins, characteristics, transport, and deposition. In Stow, D.A.V., and Piper, D.J.W. (Eds.), Fine-Grained Sediments. Geological Society Special Publications, 15(1):17-34. https://doi.org/10.1144/GSL.SP.1984.015.01.02

Manley, P.L., Pirmez, C., Busch, W., and Cramp, A., 1997. Grain-size characterization of Amazon Fan deposits and comparison to seismic facies units. In Flood, R.D., Piper, D.J.W., Klaus, A., and Peterson, L.C. (Eds.), Proceedings of the Ocean Drilling Program, Scientific Results, 155: College Station, TX (Ocean Drilling Program), 35-52. https://doi.org/10.2973/odp.proc.sr.155.209.1997 
McManus, J., 1988. Grain size determination and interpretation. In Tucker, M. (Ed.), Techniques in Sedimentology: Oxford, United Kingdom(Blackwell Science Publishing), 63-85.

Pettijohn, F.J., Potter, P.E., and Siever, R., 1987. Sand and Sandstone (2nd edition): New York (Springer-Verlag).

https://doi.org/10.1007/978-1-4612-1066-5

Shepard, F.P., 1954. Nomenclature based on sand-silt-clay ratios. Journal of Sedimentary Research, 24(3):151-158.

https://doi.org/10.1306/D4269774-2B26-11D78648000102C1865D

Shiki, T., and Yamazaki, T., 1984. A lamina-by-lamina analysis of grain-size distribution in fine-grained turbidites. Sedimentary Geology, 41(24):201-220. https://doi.org/10.1016/0037-0738(84)90062-9
Stelting, C.E., Bouma, A.H., and Stone, C.G., 2000. Fine-grained turbidite systems: overview. In Bouma, A.H., and Stone, C.G. (Eds.), Fine-Grained Turbidite Systems. AAPG Memoir, 72:1-8. https://pubs.geoscienceworld.org/books/chapter-pdf/3841038/9781629812625_ch01.pdf

Stow, D.A.V., Howell, D.G., and Nelson, C.H., 1985. Sedimentary, tectonic, and sea-level controls. In Bouma, A.H., Normark, W.R., and Barnes, N.E. (Eds.), Submarine Fans and Related Turbidite Systems: New York (Springer), 15-22. https://doi.org/10.1007/978-1-4612-5114-9_4

Stow, D.A.V., Reading, H.G., and Collinson, J.D., 1996. Deep seas. In Reading, H.G. (Ed.), Sedimentary Environments: Processes, Facies and Stratigraphy: Oxford, United Kingdom (Blackwell Science Publishing), 395-453. 